\title{
REPRESENTATIONS OF GROUPS AS QUOTIENT GROUPS. I
}

\author{
Dedicated to Hermann Weyl On His 60th Birthday \\ November 9, 1945 \\ BY \\ REINHOLD BAER
}

The classical theory of representations of a group $G$ concerns itself with some well known group $U$, like the unit group of a suitable ring or the group of all the admissible transformations of some manifold, and with all the homomorphic maps of $G$ into $U$. The theory of representations of the group $G$ which we propose to investigate here deals again with some class of well known groups $F$, like the free groups and their generalizations; but then we are concerned with the homomorphisms of these groups $F$ upon( $\left.{ }^{1}\right) G$. As a matter of fact our point of view is slightly more general. For we define: if $N$ is a normal subgroup of the group $F$, and if $F / N$ and $G$ are isomorphic groups, then $F / N$ is a representation of $G$. Well known instances of this sort of representation are the so-called "abstract definitions" of $G$ by generators and relations: in this case we impose upon a free set of generators of $F$ the relations from the normal subgroup $N$ in order to obtain $G$. These representations and those as quotient groups of free abelian groups are practically the only representations as quotient groups that have been investigated in a systematic fashion.

It is apparent that representations of groups as quotient groups and group extensions $\left({ }^{2}\right)$ are strongly related problems. For $F / N$ is a representation of $G$ if, and only if, $F$ is an extension of $N$ by $G$. These two theories are distinguished, however, by a different point of view. If we represent $G$ in the form $F / N$, then we presume that everything is known about $F$; we are not much interested in $N$ and we want information about $G$. If on the other hand we consider $F$ as an extension of $N$ by $G$, then a complete knowledge of $G$ and $N$ are presupposed; and we aim at information about $F$.

The classification of representations is the main object of this first part of our investigation. Two representations $F / M$ and $V / N$ of the group $G$ are termed equivalent whenever there exists an isomorphism of $F$ upon $V$ which maps $M$ upon $N$. Since equivalent representations are essentially identical, and since this concept of equivalence emphasizes too much the subgroups

Presented to the Society, September 17, 1945; received by the editors November 20, 1944.

(1) The word "upon" shall signify that the whole group $G$ is the image of $F$ under the homomorphism considered.

(2) For the theory of group extensions cp. Baer [1], Eilenberg-MacLane [1], Schreier [1], Zassenhaus [1]. The numbers in brackers refer to the Bibliography at the end of the paper. 
$M$ and $N$, this classification is too narrow. Thus we term the representations $F / M$ and $V / N$ of the group $G$ related representations of $G$, if there exists a homomorphism $\phi$ of $F$ into $V$ and a homomorphism $\nu$ of $V$ into $F$ such that $\phi$ and $\nu$ induce reciprocal isomorphisms between $F / M$ and $V / N$. This concept: "related representation" is reflexive, symmetric and transitive, and equivalent representations are related.

A justification for such a classification may be seen in the existence of extensive systems of invariants of classes of related representations. This is indeed the case. For we are able to derive from every representation $F / N$ of the group $G$ by means of a straightforward application of the commutator calculus various families of (inductively defined) subgroups $F(i), N(i)$ of $F$ with the following properties: (1) $F(i)$ is a fully invariant subgroup of $F$; (2) $N(i)$ is a normal subgroup of $F(i)$ and is fully invariant relative to $N$; (3) $F(i) / N(i)$ is an invariant of the class of related representations of $G$ determined by $F / N$. The third of these properties is a generalization of an invariance theorem which is due to $\mathrm{H}$. Hopf $\left({ }^{3}\right)$. If the class of related representations of $G$ is selected in some fashion independent of $G$, like the representations as quotient groups of free groups, and so on, then the invariants of this class of related representations of $G$ are actually absolute group invariants $\left({ }^{4}\right)$ of $G$.

This last remark shows the importance of finding what may be called "absolute classes of representations." We note first that a representation $F / M$ of the group $G$ reflects only in a very general way the structural properties of $G$, since it need not be possible to obtain the automorphisms of $G$ by means of endomorphisms of $F$. Hence we term $F / M$ a true representation of $G$ whenever it is possible to induce all the automorphisms of $G$ by means of endomorphisms of $F$. Two groups $F$ and $V$ shall be termed similar, provided representations $F / M$ and $V / N$ of the same group $G$ are always true as well as related. Classes of similar groups will be an appropriate tool for finding the desired absolute classes of representations, since representations of a group $G$ as quotient groups of similar groups are always both true and related. But there is a fundamental difficulty connected with this concept of similarity, since groups need not be self-similar, and since the similarity relation is not transitive. Thus it is important to obtain criteria assuring that the groups $U$ and $W$ are similar, in case both are similar to a third group $V$. Defining the concept "retract of a group" in obvious analogy to the topological concept, we may show the similarity of retracts of self-similar, similar groups. But we prove conversely that $R$ is a retract of a group in the set $\Xi$ of similar and self-similar groups if, and only if, $R$ is a homomorphic image of a group in $\Xi$ and is similar to every group in $\Xi$.

To obtain concrete instances of "absolute classes of representations" we

(3) Hopf [1]; see also Eilenberg-MacLane [3,4].

(4) For applications of these invariants, see the third part of this investigation, Baer [5]. 
prove that a group is a free group if, and only if, it is similar to every free group; and we introduce the concept of reduced free group: these are groups of the form $R=F / J$ where $J$ is a fully invariant subgroup of the free group $F$. It is noteworthy that reduced free groups admit of essentially only one representation in this normal form $F / J$; and that reduced free groups are isomorphic if each of them is a homomorphic image of the other one. If $F / M$ and $V / N$ are reduced free groups, if $F$ and $V$ are free groups, and if every homomorphism of $F$ into $V$ maps $M$ into $N$ and every homomorphism of $V$ into $F$ maps $N$ into $M$, then $F / M$ and $V / N$ are similar groups, and we term them in this case similarly reduced free groups. If $F / J$ and $V / H$ are the representations of similarly reduced free groups in normal form, then every homomorphism of $F$ into $V$ maps $J$ into $H$ and every homomorphism of $V$ into $F$ maps $H$ into $J$. Reduced free groups may be shown to be similarly reduced free groups if each of them is isomorphic to a subgroup of the other one.

If $\Xi$ is a system of similarly reduced free groups, then all the representations of a given group $G$ as quotient group of a group in $\Xi$ belong to the same class of related true representations. The invariants of these classes of representations are absolute invariants of $G$, since they depend only on $G$ and on the preassigned class $\Xi$. These classes of representations constitute therefore "absolute" classes of representations of $G$.

Classes of similarly reduced free groups are characterized by the fact that the system of their homomorphic images is the class of all groups satisfying a suitable set of identical relations $\left(^{5}\right)$. An internal characterization of these systems by properties of a lattice theoretical type, achieved in Theorem 5 of III.5, is one of the main objectives of this part of our investigation.

New results both in the theory of fully invariant subgroups and in the theory of free groups had to be obtained in order to make the necessary applications of these theories. Some of them are of interest in themselves, like the criteria for a homomorphism to be an isomorphism which we obtained in II.3 and III. 2 and the isomorphisms between lattices of fully invariant subgroups of free groups, derived in III.2.

\section{Chapter I. Derived series relative to a normal subgroup}

I.1. The commutator calculus. In this section we collect a number of definitions and facts which we shall need in the future. Though at least part of the contents of this section is well known, it will be convenient to state it in the form best suited to our purposes.

If $x$ and $y$ are any two elements in the group $G$, then their product is $x y$ and their commutator is $(x, y)=x^{-1} y^{-1} x y$. If $X$ and $Y$ are subsets of the group $G$, then $X \cap Y$ is their cross cut, $X Y$ is the subgroup generated by all the products $x y$ for $x$ in $X$ and $y$ in $Y$, and $(X, Y)$ is the subgroup $\left(^{(6)}\right.$ generated

(5) In the sense of B. Neumann [1]; see also P. Hall [2].

(6) Zassenhaus [1, p. 58]. 
by all the commutators $(x, y)$ for $x$ in $X$ and $y$ in $Y$. If $X$ and $Y$ are subgroups of $G$, then $(X, Y)=(Y, X)$, and if $X$ or $Y$ is a normal subgroup of $G$, then $X Y=Y X$.

The following three formulas are verified by direct computation $\left({ }^{7}\right)$ :

(1) $(y, x)=(x, y)^{-1}$.

(2) $(x, y z)=(x, z) z^{-1}(x, y) z=(x, z)(z,(y, x))(x, y)$.

(3) $(x y, z)=y^{-1}(x, z) y(y, z)=(x, z)((x, z), y)(y, z)$.

If $U, V$ and $W$ are groups, then it is customary to term a $W$-valued pairing operation of the groups $U$ and $V$ any binary function $u \circ v$ for $u$ in $U$ and $v$ in $V$, meeting the following requirements:

(i) $u \circ v$ is a uniquely determined element in $W$ for every $u$ in $U$ and every $v$ in $V$.

(ii) $u \circ\left(v^{\prime} v^{\prime \prime}\right)=\left(u \circ v^{\prime}\right)\left(u \circ v^{\prime \prime}\right)$.

(iii) $\left(u^{\prime} u^{\prime \prime}\right) \circ v=\left(u^{\prime} \circ v\right)\left(u^{\prime \prime} \circ v\right)$.

Theorem 1( $\left.{ }^{8}\right)$. If $M, N, H, K$ are normal subgroups of the group $G$, if $M \leqq H$ and $N \leqq K$, then a binary function of the groups $H / M$ and $K / N$ with values in $(H, K) /[(M, K)(N, H)]$ is defined by

$$
M x \circ N y \equiv(x, y) \text { modulo }(M, K)(N, H)
$$

for $x$ in $H$ and $y$ in $K$; if furthermore $(H, K) \leqq M \cap N$, then this binary function is a pairing operation.

Proof. If $m, n, h, k$ are elements in the groups $M, N, H, K$ respectively, then $k^{-1}(h, n) k$ belongs to $(N, H)$ and $h^{-1}(m, k) h$ belongs to $(M, K)$, since $(N, H)$ and $(M, K)$ are normal subgroups of $G$. Hence it follows from formulas (2) and (3) that

$$
(m h, n k) \equiv(h, k) \text { modulo }(N, H)(M, K),
$$

proving the first assertion.

Assume now that $(H, K) \leqq M \cap N$. If $u$ is in $H$ and $v^{\prime}, v^{\prime \prime}$ are in $K$, then $\left(v^{\prime}, u\right)$ is in $(H, K) \leqq M$. Hence $\left(v^{\prime \prime},\left(v^{\prime}, u\right)\right)$ belongs to $(K, M)$, and it follows from (2) that

$$
\left(u, v^{\prime} v^{\prime \prime}\right) \equiv\left(u, v^{\prime \prime}\right)\left(u, v^{\prime}\right) \text { modulo }(K, M) .
$$

Since $\left(\left(u, v^{\prime}\right),\left(u, v^{\prime \prime}\right)\right)$ belongs to $((H, K),(H, K)) \leqq(K, M)$, we deduce

$$
\left(u, v^{\prime} v^{\prime \prime}\right) \equiv\left(u, v^{\prime}\right)\left(u, v^{\prime \prime}\right) \text { modulo }(K, M) \text {. }
$$

By a slightly simpler argument one derives from (3) the congruence

$$
\left(u^{\prime} u^{\prime \prime}, v\right) \equiv\left(u^{\prime}, v\right)\left(u^{\prime \prime}, v\right) \text { modulo }(H, N)
$$

for $u^{\prime}, u^{\prime \prime}$ in $H$ and $v$ in $K$, completing the proof.

( $)$ P. Hall $[1$, p. 43$]$.

(8) The author is indebted to S. MacLane for his suggestion of substituting this more general statement for the author's original special case. 
P. Hall $\left(^{9}\right)$ has derived from (2), (3) the following fact which we state here for future reference.

LEMMA 1. If $U, V$ and $W$ are normal subgroups of the group $G$, then

$$
(U,(V, W)) \leqq(V,(W, U))(W,(U, V)) .
$$

If $S$ and $T$ are subsets of the group $G$, then we define their commutator quotient $\left({ }^{10}\right) S \div T$ as the set of all the elements $x$ in $G$ which meet the requirement : $(t, x)$ belongs to $S$ whenever $t$ is in $T$ (in symbols : $(T, x) \leqq S$ ).

Theorem 2. If $S$ and $T$ are normal subgroups of the group $G$, then $S \div T$ is the normal subgroup of $G$ which consists of all the elements in $G$ inducing the identity automorphism in $T /(S \cap T)$.

Proof. If $x$ is an element in $G$, then an automorphism of $T /(S \cap T)$ is induced by mapping the coset $(T \cap S) t$ of $T /(S \cap T)$ upon $(T \cap S) x^{-1} t x$. The elements in $G$ which induce in this fashion the identity automorphism in $T /(S \cap T)$ form a normal subgroup of $G$. Clearly the element $x$ in $G$ induces the identity automorphism in $T /(S \cap T)$ if, and only if, $(x, T) \leqq S \cap T$. But $(G, T) \leqq T$, since $T$ is a normal subgroup of $G$. Hence $(x, T) \leqq S$ if, and only if, $(x, T) \leqq S \cap T$; this completes the proof.

LemMa 2. If $R, S, T$ are normal subgroups of the group $G$, then $R \leqq S \div T$ is a necessary and sufficient condition for $T \leqq S \div R$.

The proof of this lemma is an immediate consequence of the fact that both inequalities are equivalent to $(T, R) \leqq S$.

The best known example of a commutator quotient is $1 \div G$, which is by Theorem 2 just the center $Z(G)$ of $G$. Likewise one derives from Theorem 2 that $N \leqq N \div G$ and $(N \div G) / N=Z(G / N)$ for every normal subgroup $N$ of $G$.

If $X$ and $Y$ are subsets of the group $G$, and if $\eta$ is a homomorphism of $G$, then $(X, Y)^{\eta}=\left(X^{\eta}, Y^{\eta}\right)$. It is well known that no like statement may be expected for commutator quotients $\left({ }^{11}\right)$. Thus the following result will prove useful.

THEOREM 3. If $V$ and $U \leqq V$ are normal subgroups of the group $W$, if $K$ and $H \leqq K$ are normal subgroups of the group $L$, and if $\eta$ is a homomorphism of $W$ into $L$ such that $U^{n} \leqq H$ and $V^{\eta} H=K$, then

$$
(U \div V)^{\eta} \leqq H \div K \text {. }
$$

Proof. If $x$ belongs to $U \div V$, then $(x, V) \leqq U$ and consequently $\left(x^{\eta}, V^{\eta}\right)$ $=(x, V)^{\eta} \leqq U^{\eta} \leqq H$. Since $H$ is a normal subgroup of $L$, and since $x^{\eta}$ belongs

(9) P. Hall [1, p. 47, Theorem 2.3].

(10) Zassenhaus [1].

(11) See, for instance, Baer [3, p. 145] and Levi [2, p. 92]. 
to $L$, we have furthermore $\left(x^{\eta}, H\right) \leqq H$. Hence it follows from formula (2) and the hypothesis $K=H V^{\eta}$ that $\left(x^{\eta}, K\right)=\left(x^{\eta}, H V^{\eta}\right)=\left(x^{\eta}, H\right)\left(x^{\eta}, V^{\eta}\right) \leqq H$. Consequently $x^{\eta}$ belongs to $H \div K$, as we desired to show.

Remark. $\eta$ induces, in the terminology of I.4 below, a homomorphism of $V / U$ upon $K / H$.

I.2. Definition of the $p$-derived series relative to $N$. Throughout we shall denote by $p$ an ordered pair of integral-valued functions $j(i), k(i)$ such that $0 \leqq j(i)<i$ and $0 \leqq k(i)<i$ for $0<i$. The normal subgroups $S(i)$ of $G$, for $0 \leqq i$, are said to form a $p$-derived series of $G$ if

$$
S(i) \leqq(S(j(i)), S(k(i)))
$$

for $0<i$.

There exists always a $p$-derived series. For define the subgroups $G(i)=G(i, p)$ inductively by the equations :

$$
G(0)=G, G(i)=(G(j(i)), G(k(i))) \quad \text { for } 0<i .
$$

This special $p$-derived series will always be referred to as the $p$-series of $G$. It is readily seen that the $p$-series of $G$ consists of fully invariant $\left({ }^{12}\right)$ subgroups of $G$.

Remark 1. Generalizing the concept of central series, introduced by P. Hall [1], we might say that the normal subgroups $S(i)$ of $G$ form a $p$-central series if

$$
(S(j(i)), S(k(i))) \leqq S(i) \quad \text { for } 0<i .
$$

We shall not make any use of this concept, though the $p$-series of $G$ is both $p$-derived and $p$-central.

We are not so much interested in these "absolute" invariants of the group $G$ as in some subgroups which are only "invariant relative to a distinguished normal subgroup $N$ of $G$." We define:

The ordered pairs of normal subgroups $S(i), T(i)$, for $0 \leqq i$, of the group $G$ form a p-derived series of $G$ relative to the normal subgroup $N$ of $G$, if $N \leqq S(0)$ $\leqq T(0)$ and if, for positive $i$,

$$
(S(j(i)), T(k(i)))(S(k(i)), T(j(i))) \leqq S(i) \leqq T(i) \leqq(T(j(i)), T(k(i))) .
$$

Thus the $T(i)$ alone form a $p$-derived series of $G$.

There exists always a $p$-derived series of $G$ relative to $N$, namely the following inductively defined series of pairs of subgroups $N(i)=N(i, p)$, $G(i)=G(i, p)$ whose defining equations are :

$$
\begin{aligned}
N(0) & =N, \quad G(0)=G, & & \\
N(i) & =(N(j(i)), G(k(i)))(N(k(i)), G(j(i))) & & \text { for } 0<i, \\
G(i) & =(G(j(i)), G(k(i))) & & \text { for } 0<i .
\end{aligned}
$$

(12) A subgroup is fully invariant if it is mapped into itself by every endomorphism of the group. Endomorphisms are homomorphisms of a group into itself. 
This special $p$-derived series relative to $N$ will always be referred to as the $p$-series relative to $N$ or the $p$-N-series of $G$. It is readily verified that the $p-N$-series of $G$ is fully invariant relative to $N$ in the sense that every endomorphism of $G$ which maps $N$ into part of $N$ maps at the same time $N(i)$ into part of $N(i)$ and $G(i)$ into part of $G(i)$, and one may prove inductively $N(i) \leqq N$.

THEOREM 1. If a p-derived series of $G$ relative to the normal subgroup $N$ of $G$ is formed by the pairs $S(i), T(i)$ of normal subgroups of $G$, then

(1) $T(i) \leqq G(i, p)$ for $0 \leqq i$;

(2) $T(i)=G(i)$, for every $i$, implies $N(i, p) \leqq S(i)$ for $0 \leqq i$;

(3) $N \leqq N \div G \leqq(S(j(i)) \div T(j(i))) \cap(S(k(i)) \div T(k(i))) \leqq S(i) \div T(i)$;

(4) $N(i, p) \leqq S(i)$, for every $i$, implies $N(i) \div G(i) \leqq S(i) \div T(i)$ for $0 \leqq i$.

Remark 2. If in particular $j(i)=k(i)=i-1$ for positive $i$, then we deduce from (3) that $S(i-1) \div T(i-1) \leqq S(i) \div T(i)$ for positive $i$.

Proof. Clearly $T(0) \leqq G(0)$, since $G(0, p)=G$. Using induction and the definition of a $p$-derived series (relative to $N$ ) we find that proving (1).

$$
T(i) \leqq(T(j(i)), T(k(i))) \leqq(G(j(i)), G(k(i)))=G(i),
$$

Clearly $N(0) \leqq S(0)$, since $N(0)=N$. Using induction, the definitions, and the additional hypothesis $T(i)=G(i)$ we find

proving (2).

$$
\begin{aligned}
N(i) & =(N(j(i)), G(k(i)))(N(k(i)), G(j(i))) \\
& \leqq(S(j(i)), T(k(i)))(S(k(i)), T(j(i))) \leqq S(i),
\end{aligned}
$$

The normality of the subgroup $N$ of $G$ is equivalent to the inequality $(G, N) \leqq N$, and this inequality is equivalent to $N \leqq N \div G$. For positive $i$ we put $D(i)=(S(j(i)) \div T(j(i))) \cap(S(k(i)) \div T(k(i)))$. Then we deduce from the definition of commutator quotients that $(T(j(i)), D(i)) \leqq S(j(i))$ and $(T(k(i)), D(i)) \leqq S(k(i))$. Applying the definition of a $p$-derived series (relative to $N$ ) and Lemma 1 of $I .1$ we find that

$$
\begin{aligned}
(T(i), D(i)) & \leqq((T(j(i)), T(k(i))), D(i)) \\
& \leqq(T(j(i)),(T(k(i)), D(i)))(T(k(i)),(T(j(i)), D(i))) \\
& \leqq(T(j(i)), S(k(i)))(T(k(i)), S(j(i))) \leqq S(i)
\end{aligned}
$$

or $D(i) \leqq S(i) \div T(i)$. From $N \leqq S(0)$ one deduces that $N \div G \leqq S(0) \div T(0)$. Hence it follows inductively that $N \div G \leqq D(i) \leqq S(i) \div T(i)$ so that $N \div G$ $\leqq S(i) \div T(i)$ holds for every $i$. This completes the proof of (3). (4) is an almost immediate consequence of (1) and its special hypothesis.

If $p=(j(i), k(i))$ is an admissible pair of integral-valued functions, then we have in particular $j(1)=k(1)=0$. Consequently

$$
N(0, p)=N, \quad G(0, p)=G ; \quad N(1, p)=(N, G), \quad G(1, p)=(G, G) .
$$


Thus the first two pairs of all $p-N$-series are the same. We shall indicate later possibilities of generalizing the conditions imposed upon these series in such a way that the range of the first two terms is extended substantially. The following remark will be of some interest.

Corollary. If $N$ is a normal subgroup of $G$, then $(N \div G) / N$ is the center of $G / N,[N \div(G, G)] / N$ is the centralizer $\left.{ }^{13}\right)$ of the commutator subgroup of $G / N$ in $G / N$, and $N \div G \leqq(N, G) \div(G, G) \leqq N \div(G, G)$.

This is an almost immediate inference from (3) of Theorem 1 and the inequality $(N, G) \leqq N$ which is valid for every normal subgroup $N$ of $G$.

We shall be particularly interested in a special class of series of the type described in this section. The ordered pairs of normal subgroups $S(i), T(i)$, for $0 \leqq i$, of the group $G$ are said to form an interlocking series, if $T(0)=G$ and $S(i+1) \leqq S(i) \leqq T(i+1) \leqq T(i)$ for $0 \leqq i$. It is termed strictly interlocking, if in addition 1 is the cross cut of the subgroups $S(i)$.

THEOREM 2. If the subgroups $S(i), T(i)$ form an interlocking series, if $U$ is a subset of $G$ such that

$$
U \cap S(i)=U \cap T(i)
$$$$
\text { for } 0 \leqq i \text {, }
$$

then $U$ is part of the cross cut $S(\omega)$ of all the subgroups $S(i)$.

Proof. For $i=0$ we infer from our hypothesis that

$$
U=U \cap G=U \cap T(0)=U \cap S(0) \text { or } U \leqq S(0) \text {. }
$$

Suppose that we have already verified $U \leqq S(i)$. Since the series is interlocking, this implies $U \leqq T(i+1)$, and hence it follows from the hypothesis that $U=U \cap T(i+1)=U \cap S(i+1)$ or $U \leqq S(i+1)$. Thus $U \leqq S(\omega)$ is proved by complete induction.

THEOREM 3. If the pairs of normal subgroups $S(i), T(i)$ of the group $G$ form an interlocking $p$-derived series of $G$ relative to its normal subgroup $N$, then $S(i-1) / S(i) \leqq Z(T(i-1) / S(i))$.

Here $Z(H)$ indicates the center $1: H$ of the group $H$.

Proof. Both the series $S(i)$ and $T(i)$ are monotone decreasing. Hence $S(i-1) \leqq S(h), T(i-1) \leqq T(h)$ for $h=j(i)$ or $k(i)$. Furthermore

$$
(S(i-1), T(i-1)) \leqq(S(j(i)), T(k(i)))(S(k(i)), T(j(i))) \leqq S(i),
$$

and this proves our contention. (Note that we did not make use of the condition $S(i-1) \leqq T(i)$ !)

I.3. The derived and the lower central series relative to $N$. Of the $p-N$ series of a group $G$, two are of particular importance. Thus we shall attempt to obtain more precise results for these special series.

(13) If $S$ is a subgroup of the group $G$, then the centralizer of $S$ in $G$ is formed by all the elements $z$ in $G$ which satisfy $z s=s z$ for every $s$ in $S$. 
The derived series relative to the normal subgroup $N$ of $G$. Its members are denoted by $N_{i}, G^{(i)}$, and they are defined inductively by the equations

$$
N_{0}=N, G^{(0)}=G \quad \text { and } N_{i}=\left(G^{(i-1)}, N_{i-1}\right), G^{(i)}=\left(G^{(i-1)}, G^{(i-1)}\right)
$$

for positive $i$. Clearly $N_{i}$ is part of $G^{(i)}$, and both the $N_{i}$ and the $G^{(i)}$ form a descending chain of normal subgroups of $G$. If we put $j(i)=k(i)=i-1$, for positive $i$, then the derived series relative to $N$ is obviously just the $p-N$ series of $G$ for this particular choice of the pair $p$.

If $0 \leqq m<n$, then $G^{(n-1)} \leqq G^{(m)}$ and therefore $\left(N_{m}, G^{(n-1)}\right) \leqq\left(N_{m}, G^{(m)}\right)$ $=N_{m+1}$. Furthermore $N_{n-1} \leqq N_{m}$ and hence $\left(N_{n-1}, G^{(m)}\right) \leqq\left(N_{m}, G^{(m)}\right)=N_{m+1}$. Using the commutator quotients these inequalities may be restated in the following fashion.

$$
\begin{aligned}
& G^{(n-1)} \leqq N_{m+1} \div N_{m}, \quad G^{(m)} \leqq N_{m+1} \div N_{n-1}, \\
& N_{n-1} \leqq N_{m+1} \div G^{(m)}, \quad N_{m} \leqq N_{m+1} \div G^{(n-1)} \text {. }
\end{aligned}
$$

To enunciate the following slightly deeper property of this series we need the ascending (or upper) central chain $Z_{i}(H)$ of the group $H$ which may be defined inductively as follows:

$$
Z_{0}(H)=1, \quad Z_{i}(H)=Z_{i-1}(H) \div H \quad \text { for positive } i .
$$

It is readily seen that $Z_{i}(H) / Z_{i-1}(H)$ is just the center of $H / Z_{i-1}(H)$ so that this definition coincides with the usual definition of this chain(14).

THEOREM 1. If $N$ is a normal subgroup of $G$, and if $0 \leqq i \leqq n$, then

$$
\left(G^{(n)} \cap N_{n-i}\right) / N_{n} \leqq Z_{i}\left(G^{(n)} / N_{n}\right) .
$$

Proof. The inequality under consideration is certainly true for $i=0$; and thus we may assume its validity for $i-1$ in order to prove it for $i$. Applying the fact that the derived series is descending and making use of the last of the four inequalities (1), we show:

$$
\begin{aligned}
G^{(n)} \cap N_{n-i} & \leqq G^{(n)} \cap\left(N_{n-i+1} \div G^{(n-1)}\right) \leqq G^{(n)} \cap\left(N_{n-i+1} \div G^{(n)}\right) \\
& \leqq G^{(n)} \cap\left\{\left(x, G^{(n)}\right) \leqq N_{n-i+1}\right\} \\
& \leqq\left\{\left(x, G^{(n)}\right) \leqq\left(N_{n-i+1} \cap\left(G^{(n)}, G^{(n)}\right)\right)\right\} \\
& \leqq\left\{\left(x, G^{(n)}\right) \leqq\left(N_{n-i+1} \cap G^{(n)}\right)\right\}=\left(N_{n-i+1} \cap G^{(n)}\right) \div G^{(n)}
\end{aligned}
$$

Now we deduce from the induction hypothesis that

completing the proof.

$$
\begin{aligned}
\left(G^{(n)} \cap N_{n-i}\right) / N_{n} & \leqq\left[\left(N_{n-i+1} \cap G^{(n)}\right) \div G^{(n)}\right] / N_{n} \\
& \leqq\left[\left(N_{n-i+1} \cap G^{(n)}\right) / N_{n}\right] \div\left[G^{(n)} / N_{n}\right] \\
& \leqq Z_{i-1}\left(G^{(n)} / N_{n}\right) \div\left(G^{(n)} / N_{n}\right)=Z_{i}\left(G^{(n)} / N_{n}\right),
\end{aligned}
$$

(i4) See for instance Zassenhaus [1, p. 44]. 
Theorem 2. If $N$ is a normal subgroup of $G$, then

$$
N_{i} \leqq N(i, p) \text { and } G^{(i)} \leqq G(i ; p) \quad \text { for every } i \text { and } p .
$$

Proof. From the fact that both the $N_{i}$ and the $G^{(i)}$ are monotone decreasing and that $j(i)<i, k(i)<i$, we deduce by complete induction

$$
\begin{aligned}
N_{i} & =\left(N_{i-1}, G^{(i-1)}\right) \leqq\left(N_{j(i)}, G^{(k(i))}\right)\left(N_{k(i)}, G^{(i(i))}\right) \\
& \leqq(N(j(i), p), G(k(i), p))(N(k(i), p), G(j(i), p))=N(i, p), \\
G^{(i)} & =\left(G^{(i-1)}, G^{(i-1)}\right) \leqq\left(G^{(j(i))}, G^{(k(i))}\right) \\
& \leqq(G(j(i), p), G(k(i), p))=G(i, p),
\end{aligned}
$$

proving our contention.

The lower central series relative to the normal subgroup $N$ of $G$. Its members are denoted by ${ }_{i} N,{ }^{i} G$; and they are defined inductively by the equations:

$$
{ }_{0} N=N,{ }^{\circ} G=G \text { and }{ }_{i} N=\left(G,{ }_{i-1} N\right),{ }^{i} G=\left(G,{ }^{i-1} G\right) \text { for positive } i .
$$

Clearly ${ }_{i} N$ is part of ${ }^{i} G$; and both the ${ }_{i} N$ and the ${ }^{i} G$ form a descending chain of normal subgroups of $G$. The following two inequalities are readily verified by complete induction and application of Lemma 1 of I.1.

$$
\left({ }^{i} G,{ }_{j} N\right) \leqq{ }_{1+i+j} N \text { and } N_{i} \varliminf_{26-1} N .
$$

Choosing in particular $N=G$ we obtain the following important special case of (2).

$$
\left({ }^{i} G,{ }^{i} G\right) \leqq{ }^{1+i+i} G \text { and } G^{(i)} \leqq{ }^{2 i-1} G .
$$

Denote by ${ }_{\omega} N$ the cross cut of the subgroups ${ }_{i} N$ and by ${ }^{\omega} G$ the cross cut of the subgroups ${ }^{i} G$.

$$
\left({ }^{\omega} G, N\right) \leqq{ }_{\omega} N \leqq{ }^{\omega} G .
$$

Proof. Clearly ${ }_{\omega} N \leqq_{i} N \leqq^{i} G$ for every $i$, proving ${ }_{\omega} N \leqq{ }^{\omega} G$. Furthermore it follows from (2) that

$$
\left({ }^{(} G, N\right) \leqq\left({ }^{i} G, N\right) \leqq{ }_{i+1} N
$$

for every $i$, and hence $\left({ }^{\omega} G, N\right) \leqq{ }_{\omega} N$.

Using the concept of commutator quotient we may restate the first inequality (2) in two different forms:

$$
{ }_{n-m-1} N \leqq{ }_{n} N \div{ }^{m} G \text { and }{ }^{n-m-1} G \leqq{ }_{n} N \div{ }_{m} N \quad \text { for } 0 \leqq m<n .
$$

A special case of $(2)$ is the inequality $\left({ }^{i} G, N\right) \leqq_{1+i} N=\left(G,{ }_{i} N\right)$. But this shows that the lower central series relative to $N$ is just the $p$ - $N$-series obtained by selecting $j(i)=0, k(i)=i-1$.

(15) For a detailed proof see Zassenhaus [1, p. 120]. 
THEOREM 3. The lower central series of the group $G$ relative to its normal subgroup $N$ is interlocking, if (and only if) $N \leqq(G, G)$.

Proof. By hypothesis we have $0 N=N \leqq(G, G)={ }^{1} G$. Thus we may assume that we have already verified ${ }_{i-1} N \leqq{ }^{i} G$ for some positive $i$. Then ${ }_{i} N=\left(G,{ }_{i-1} N\right) \leqq\left(G,{ }^{i} G\right)={ }^{i+1} G$, proving our contention.

Remark. If 1 happens to be the cross cut of the subgroups ${ }^{i} G$, a property which is, for example, enjoyed by the free groups $\left({ }^{16}\right)$, then the lower central series of $G$ relative to $N$ is strictly interlocking, provided $N \leqq(G, G)$.

For a more detailed investigation of the lower central series relative to $N$ see the second part of this investigation $\left({ }^{17}\right)$.

I.4. Invariance of $p$ - $N$-series. If $S, T$ are subgroups of the group $G$, if $S$ is a normal subgroup of $T$, and if the endomorphism $\eta$ of $G$ satisfies $t^{\eta} \equiv t$ modulo $S$ for every $t$ in $T$, then $\eta$ may be said to induce the identity in $T / S$. If $\eta$ induces the identity in $T / S$, then $S^{n} \leqq S$ and $T^{n} S=T$.

THEOREM 1. If the normal subgroups $S(i), T(i)$ form a p-derived series of the group $G$ relative to the normal subgroup $N$ of $G$, and if the endomorphism $\eta$ of $G$ induces the identity in $G / N$, then $\eta$ induces the identity in every $T(i) / S(i)$ for $0 \leqq i$.

Proof. The endomorphism $\eta$ induces the identity in $T(0) / S(0)$, since $N \leqq S(0) \leqq T(0)$, and since $\eta$ induces the identity in $G / N$. Hence we may assume that the identity is induced by $\eta$ in every $T(h) / S(h)$ for $0 \leqq h<i$, in order to prove that the identity is induced by $\eta$ in $T(i) / S(i)$. It follows from this induction hypothesis that the identity is induced by $\eta$ in $T(j(i)) / S(j(i))$ as well as in $T(k(i)) / S(k(i))$. If $n$ is either $j(i)$ or $k(i)$, and if $t$ is an element in $T(n)$, then we have therefore $t \eta \equiv t$ modulo $S(n)$. Hence we deduce from the formulas (2), (3) of I.1 or from Theorem 1 of I.1 that for $x$ in $T(j(i))$ and $y$ in $T(k(i))$

$$
(x, y)^{\eta}=\left(x^{\eta}, y^{\eta}\right) \equiv(x, y) \text { modulo }(S(j(i)), T(k(i)))(S(k(i)), T(j(i))) .
$$

But $(T(j(i)), T(k(i)))$ is generated by these commutators $(x, y)$. Thus it follows that the identity is induced by $\eta$ in

$$
(T(j(i)), T(k(i))) /[(S(j(i)), T(k(i)))(S(k(i)), T(j(i)))],
$$

and this implies in particular that the identity is induced by $\eta$ in $T(i) / S(i)$, as follows from the definition of a $p$-derived series relative to $N$ (as given in I.2).

Remark. This theorem admits of various generalizations and each generalization leads naturally to a corresponding extension of the subsequent invariance theorems whose proofs are based on Theorem 1 . We do not attempt

(16) Magnus [1].

(17) Baer [4]. 
here to reach the acme of generality $\left({ }^{18}\right)$ and thus we content ourselves with giving a typical example.

If $S$ is a subgroup of the group $G$, then we denote by $S^{n}$ the subgroup of $S$ which is generated by all the $n$th powers of elements in $S$. If $N$ is a normal subgroup of $G$, then $N /(N, G)$ is part of the center of $G /(N, G)$. Hence

$$
(x y)^{n} \equiv x^{n} y^{n} \text { modulo }(N, G) \quad \text { for } x \text { in } G \text { and } y \text { in } N \text {. }
$$

If the endomorphism $\eta$ of $G$ induces the identity in $G / N$, then it follows now that the identity is induced by $\eta$ in $\left[G^{n}(G, N)\right] /\left[N^{n}(G, N)\right]$. If we combine this result with Theorem 1 , then we find that the identity is induced by $\eta$ in $\left[G^{n}(G, G)\right] /\left[N^{n}(G, N)\right]$.

If $M$ and $N$ are normal subgroups of the groups $H$ and $K$ respectively, if $\eta$ is a homomorphism of $H$ into $K$, and if $X$ is a coset of $H / M$, then $X$ " is a subset of $K ; X^{\eta}$ is part of one coset of $K / N$ if, and only if, $M^{\eta} \leqq N$. If $M$ is mapped by $\eta$ upon part of $N$, then the coset which contains $X^{\eta}$ consists of all the products $n x \eta$ for $n$ in $N$ and $x$ in $X$, and thus we may denote this coset without danger of confusion by $N X^{*}$. If $M^{*} \leqq N$, then a homomorphism of $H / M$ into $K / N$ is defined by mapping the coset $X$ of $H / M$ upon the coset $N X^{n}$ of $K / N$. This homomorphism shall always be termed the homomorphism induced by $\eta$, and whenever we speak of the homomorphism (or isomorphism) of $H / M$ into $K / N$ which is induced by $\eta$, this shall be supposed to imply $M^{*} \leqq N$. We are now ready to state and prove the main result of this section.

Theorem 2. If $M$ and $N$ are normal subgroups of the groups $H$ and $K$ respectively, if the isomorphism $\alpha$ of $H / M$ upon $K / N$ is induced by the homomorphism $\eta$ of $H$ into $K$, and if $\alpha^{-1}$ is induced by the homomorphism $\kappa$ of $K$ into $H$, then an isomorphism $\alpha(i)$, for $0 \leqq i$, of $H(i, p) / M(i, p)$ upon $K(i, p) / N(i, p)$ is induced by $\eta$ and $\alpha(i)^{-1}$ is induced by $k$.

Note that the theorem holds for every admissible pair $p$.

Proof. It is readily verified by complete induction (and has been pointed out before) that $H(i, p)^{\eta} \leqq K(i, p), K(i, p)^{\kappa} \leqq H(i, p)$ and $M(i, p)^{n} \leqq N(i, p)$, $N(i, p)^{\kappa} \leqq M(i, p)$, and that therefore a homomorphism $\alpha(i)$ of $H(i, p) / M(i, p)$ into $K(i, p) / N(i, p)$ is induced by $\eta$ and a homomorphism $\beta(i)$ of $K(i, p) / N(i, p)$ into $H(i, p) / M(i, p)$ is induced by $\kappa$. It is an immediate consequence of our hypothesis that $\eta \kappa$ is an endomorphism of $H$ which induces the identity in $H / M$. Hence it follows from Theorem 1 that $\eta \kappa$ induces the identity in every $H(i, p) / M(i, p)$. But $\alpha(i) \beta(i)$ is induced by $\eta \kappa$ in $H(i, p) / M(i, p)$, and thus it follows that $\alpha(i) \beta(i)=1$. Likewise one proves $\beta(i) \alpha(i)=1$, and this makes our contention evident.

LEMMA. If $R$ and $S$ are normal subgroups of the groups $U$ and $V$ respectively, [1].

(18) $\mathrm{Cp}$. in this context the theory of marginal and word subgroups. P. Hall [2], Neumann 
if the isomorphism $\alpha$ of $U / R$ upon $V / S$ is induced by the homomorphism $\eta$ of $U$ into $V$ and the isomorphism $\alpha^{-1}$ is induced by the homomorphism $\alpha$ of $V$ into $U$, and if $A$ and $B$ are subgroups such that $R \leqq A \leqq U, S \leqq B \leqq V, A^{*} \leqq B, B^{*} \leqq A$, then

$$
(A / R)^{\alpha}=B / S \text {. }
$$

Proof. One deduces readily from the hypotheses that

$$
B / S=(B / S)^{\alpha^{-1} \alpha}=\left(\left(B^{\star} R\right) / R\right)^{\alpha} \leqq(A / R)^{\alpha}=\left(A^{\eta} S\right) / S \leqq B / S,
$$

and the desired equality is an immediate consequence of these inequalities.

Using the hypotheses and notations of Theorem 2 one readily deduces the following equations from Theorem 2, the above Lemma, Theorem 3 of I.1, and Theorem 1 of I.2.

$$
\begin{gathered}
{[(M(i, p) \div H(i, p)) / M]^{\alpha}=(N(i, p) \div K(i, p)) / N} \\
{[(H(i, p) \cap H(m, p)) M(i, p) / M(i, p)]^{\alpha(i)}} \\
\quad=(K(i, p) \cap K(m, p)) N(i, p) / N(i, p) \\
{[(H(i, p) \cap M(m, p)) M(i, p) / M(i, p)]^{\alpha(i)}} \\
=(K(i, p) \cap N(m, p)) N(i, p) / N(i, p) .
\end{gathered}
$$

Since $M(i) \leqq H(i)$, we may deduce from Dedekind's law that

$$
(H(i, p) \cap H(m, p)) M(i, p)=H(i, p) \cap H(m, p) M(i, p)
$$

and

$$
(H(i, p) \cap M(m, p)) M(i, p)=H(i, p) \cap M(m, p) M(i, p),
$$

and by means of these equations various simplifications of formulas (2), (3) are possible in special instances. Of these we mention only the following one which will be most important for our applications.

(4) If the $p-M$-series of $H$ and the $p$ - $N$-series of $K$ are both interlocking, then $[M(i-1, p) / M(i, p)]^{\alpha(i)}=N(i-1, p) / N(i, p)$.

The commutativity of these quotient groups is a consequence of Theorem 3 of I.2.

I.5. A general isomorphism principle. It is the object of this section to derive certain criteria for a homomorphism to induce an isomorphism in certain quotient groups, provided it is known that this homomorphism induces isomorphisms in certain other quotient groups. The basic criterion is the following simple fact.

Lemma 1. If $M$ and $N$ are normal subgroups of the groups $H$ and $K$ respectively, and if the homomorphism $\eta$ of $H$ into $K$ induces an isomorphism of $M$ upon $N$ and an isomorphism of $H / M$ upon $K / N$, then $\eta$ is an isomorphism of $H$ upon $K$.

(19) This admits of various generalizations. 
Proof. If $x^{\eta}=1$ for $x$ in $H$, then $N=N(M x)^{\eta}$ and hence $M x=M$, since $\eta$ induces an isomorphism of $H / M$. Consequently $x$ is in $M$. But this implies $x=1$, since $\eta$ induces an isomorphism in $M$. Thus $\eta$ has been shown to be an isomorphism of $H$.

If $y$ is an element in $K$, then there exists an element $z$ in $H$ such that $z$ " is in $N y$, since $\eta$ induces an isomorphism of $H / M$ upon $K / N$. Hence $y\left(z^{\eta}\right)^{-1}$ is an element $r$ in $N$. But $\eta$ effects an isomorphism of $M$ upon $N$, and hence there exists an element $s$ in $M$ such that $s^{\eta}=r$. Thus $y=r z^{\eta}=s^{\eta} z^{\eta}=(s z) \eta$, proving $H^{\eta}=K$.

Remark. It should be noted that $\eta$ is not uniquely determined by the isomorphisms it induces in $M$ and $H / M$, since there exist, in general, automorphisms of $H$ which are different from the identity, though they induce the identity both in $M$ and in $H / M$.

If $P(i)$, for $0 \leqq i$, is a descending chain of normal subgroups of the group $Q$, then the derived group

$$
\overline{(Q ; P(i))}
$$

may be defined as follows: its elements may be represented in one and only one way in the form $x=(x(0), \cdots, x(i), \cdots)$ where $x(i)$ is a coset of $Q / P(i)$ and where $x(i) \leqq x(i-1)$ for positive $i$; the product of the elements $x$ and $y$ is obtained in the customary fashion as

$$
x y=(x(0) y(0), \cdots, x(i) y(i), \cdots) .
$$

If $P(\omega)$ is the cross cut of the subgroups $P(i)$, then it is readily seen that

$$
\overline{(Q ; P(i))}
$$

depends on $Q / P(\omega)$ only and that the elements of $Q / P(\omega)$ are "everywhere dense in

$$
\overline{(Q ; P(i))} . "
$$

Lemma 2. If $M(i)$ is a descending chain of normal subgroups of $M(0)=H$, if $N(i)$ is a descending chain of normal subgroups of $N(0)=K$, and if the homomorphism $\eta$ of $H$ into $K$ induces, for every $i$, an isomorphism of $M(i-1) / M(i)$ upon $N(i-1) / N(i)$, then:

(a) $\eta$ induces an isomorphism of $H / M(i)$ upon $K / N(i)$.

(b) $\eta$ induces an isomorphism of $H / M(\omega)$ into $K / N(\omega)$.

(c) $\eta$ induces an isomorphism of

$$
\overline{(H ; M(i))} \text { upon } \overline{(K ; N(i))} \text {. }
$$

Proof. Statement (a) is immediately deduced by complete induction from Lemma 1.

$M(\omega)^{n} \leqq N(\omega)$, since $M(i)^{n} \leqq N(i)$ by hypothesis; and thus $\eta$ induces a 
homomorphism of $H / M(\omega)$ into $K / N(\omega)$. If the element $x$ in $H$ is mapped by $\eta$ upon an element in $N(\omega)$, then $N(i)(M(i) x)^{\eta}=N(i) x^{\eta}=N(i)$ for every $i$. Hence $M(i)=M(i) x$ for every $i$, proving that $x$ belongs to every $M(i)$ and therefore to $M(\omega)$. Thus $\eta$ induces an isomorphism of $H / M(\omega)$ into $K / N(\omega)$.

If $x=(x(0), \cdots, x(i), \cdots)$ is an element in

$$
\overline{(H ; M(i))} \text {, }
$$

then we define $x^{\beta}=(x(0) \eta, \cdots, x(i) \eta, \cdots)$, and this function $\beta$ is readily seen to be a homomorphism of

$$
\overline{(H ; M(i))} \text { into } \overline{(K ; N(i))} \text {, }
$$

since an isomorphism of $H / M(i)$ upon $K / N(i)$ is induced by $\eta$ (statement (a)!). This last fact clearly implies that $x^{\beta}=y^{\beta}$ if, and only if, $x(i)=y(i)$ for every $i$, proving that $\beta$ is an isomorphism. If finally $z$ is some element in

$$
\overline{(K ; N(i))} \text {, }
$$

then there exists, for every $i$, one and only one coset $r(i)$ of $H / M(i)$ such that $z(i)=N(i) r(i)^{\eta}$. Then

$$
\begin{aligned}
{[M(i-1) r(i)] \eta } & =M(i-1) \eta r(i) \eta \leqq N(i-1) z(i) \\
& \leqq N(i-1) z(i-1)=z(i-1) .
\end{aligned}
$$

But $M(i-1) r(i)$ is a coset of $H / M(i-1)$ and $r(i-1)$ is the only coset of $H / M(i-1)$ which is mapped by $\eta$ into part of $z(i-1)$. Hence $r(i-1)$ $=M(i-1) r(i)$ or $r(i) \leqq r(i-1)$. Thus $r=(\cdots, r(i), \cdots)$ is a well determined element of

$$
\overline{(H ; M(i))}
$$

such that $\gamma^{\beta}=z$. Hence $\beta$ is an isomorphism of

$$
\overline{(H ; M(i))} \text { upon } \overline{(K ; N(i))} \text {, }
$$

completing the proof.

\section{Chapter II. Similarity}

II.1. Classification of representations. If $M$ is a normal subgroup of the group $H$, and if $H / M$ is isomorphic to the group $G$, then we consider $H / M$ as a representation of the group $G$. The two representations $H / M$ and $K / N$ of the same group $G$ may be called equivalent if there exists an isomorphism of $H$ upon $K$ which maps $M$ upon $N$. But this classification of representations, though quite natural, is too narrow for our purposes. Thus we say that the two representations $H / M$ and $K / N$ of the same group $G$ are related if there exists a homomorphism $\eta$ of $H$ into $K$ and a homomorphism $\kappa$ of $K$ into $H$ with the following property: 
(R) $\eta$ induces an isomorphism $\alpha$ of $H / M$ upon $K / N$ and $\kappa$ induces the isomorphism $\alpha^{-1}$ of $K / N$ upon $H / M$.

It is readily verified that this concept of relatedness is reflexive, symmetric and transitive, and that equivalent representations are related.

For many of our purposes the concept of representation is too general. The representation $H / M$ of the group $G$ shall be termed a true representation if there exists to every automorphism of $H / M$ an endomorphism of $H$ inducing it in $H / M$. The validity of the following statement is immediately obvious.

The representations $H / M$ and $K / N$ of the same group $G$ are related true representations if, and only if, they meet the following requirement.

(S) To every isomorphism $\alpha$ of $H / M$ upon $K / N$ there exists a homomorphism $\eta$ of $H$ into $K$ inducing $\alpha$; to every isomorphism $\beta$ of $K / N$ upon $H / M$ there exists $a$ homomorphism $\alpha$ of $K$ into $H$ inducing $\beta$.

It will be convenient to term related true representations, that is, representations meeting requirement (S), similar representations. This concept of similarity is certainly symmetric and transitive, but it need not be reflexive, since only true representations are self-similar.

There exists always a true representation of the group $G$, namely the representation $G / 1$ of $G$. We term this representation the trivial representation of $G$, and we prove as a first justification of our terminology the following fact.

THEOREM. The representation $H / M$ of the group $G$ is similar to the trivial representation if, and only if, $H$ is a splitting extension of $M$ by $G$.

The extension $H$ of $M$ by $G$ is said to be splitting if there exists a subgroup of $H$ which contains one and only one element of every coset of $H / M$.

Proof. Clearly every isomorphism of $H / M$ upon $G$ is induced by a homomorphism of $H$ upon $G$. Suppose now that $H$ is a splitting extension of $M$ by $G$. Then there exists a subgroup $S$ of $H$ which contains one and only one element in every coset of $H / M$. If $\alpha$ is an isomorphism of $G$ upon $H / M$, $x$ an element in $G$, then there exists one and only one element $x^{*}$ in the cross cut of $S$ and $x^{\alpha}$. Mapping $x$ upon $x^{*}$ is an isomorphism of $G$ into $H$ which induces $\alpha$. Suppose finally that $H / M$ and $G / 1$ are similar representations of $G$. Then there exists a homomorphism $\gamma$ of $G$ into $H$ which induces an isomorphism between $G$ and $H / M$. Consequently $G^{\gamma}$ is a subgroup of $H$ which contains one and only one element of every coset of $H / M$.

Note that in the proof of the necessity of our condition we only had to assume that $H / M$ and $G / 1$ are related representations.

It is now readily verified that all representations of the group $G$ are similar (or related) if, and only if, $G$ is a free group. To prove this one has to remember that every group may be represented as a quotient group of a free group, that subgroups of free groups are free groups (Schreier's Theorem) $\left({ }^{20}\right)$, and

$\left.{ }^{(20}\right)$ Schreier [1]; for further proofs see, for example, Levi [1] where further references may be found. 
that extensions by free groups are splitting extensions $\left({ }^{21}\right)$.

The free groups are, however, not the only groups all of whose representations are true representations. A group $G$ has this property, for example, if every automorphism of $G$ is an inner automorphism, a property which is satisfied by all the finite and infinite symmetric groups of degree greater than 6 .

If on the other hand the group $G$ is the direct product of two isomorphic groups $G^{\prime}$ and $G^{\prime \prime}$ which are not free groups, and if $H^{\prime} / M$ is a representation of $G^{\prime}$ by means of a free group $H^{\prime}$, then the representation $\left(H^{\prime} \times G^{\prime \prime}\right) / M$ of $G$ is readily seen not to be a true representation of $G$.

II.2. Invariants of classes of related representations. Suppose that $M$, $S^{\prime}, S^{\prime \prime}$ are normal subgroups of $H, S^{\prime} \leqq S^{\prime \prime}$, that $N, T^{\prime}, T^{\prime \prime}$ are normal subgroups of $K, T^{\prime} \leqq T^{\prime \prime}$, that $\alpha$ is an isomorphism of $H / M$ upon $K / N$ and that $\beta$ is an isomorphism of $S^{\prime \prime} / S^{\prime}$ upon $T^{\prime \prime} / T^{\prime}$. Then $\beta$ is said to be induced by $\alpha$, if $\beta$ is the (one and) only isomorphism of $S^{\prime \prime} / S^{\prime}$ upon $T^{\prime \prime} / T^{\prime}$ meeting the following requirement.

(I) There exists a homomorphism $\eta$ of $H$ into $K$ which induces $\alpha$ in $H / M$ and $\beta$ in $S^{\prime \prime} / S^{\prime}$.

Note that this concept of inducing an isomorphism coincides with the usual one, in case $S^{\prime \prime} / S^{\prime}$ is a sub-quotient-group of $H / M$.

THEOREM 1. (a) If $H / M$ and $K / N$ are related representations of the same group $G$, then there exists an isomorphism $\alpha$ of $H / M$ upon $K / N$ which induces an isomorphism $\alpha(i, p)$ of $H(i, p) / M(i, p)$ upon $K(i, p) / N(i, p)$ (for every $i$ and $p$ ).

(b) If $H / M$ and $K / N$ are similar representations of the same group $G$, then every isomorphism $\alpha$ of $H / M$ upon $K / N$ induces, for every $i$ and $p$, an isomorphism $\left({ }^{22}\right) \alpha(i, p)$ of $H(i, p) / M(i, p)$ upon $K(i, p) / N(i, p)$.

Proof. If the isomorphism $\alpha$ of $H / M$ upon $K / N$ is induced by the homomorphism $\eta$ of $H$ into $K$ and the isomorphism $\alpha^{-1}$ by the homomorphism $\kappa$ of $K$ into $H$, then we deduce from Theorem 2 of $I .4$ that an isomorphism $\alpha(i, p)$ of $H(i, p) / M(i, p)$ upon $K(i, p) / N(i, p)$ is induced by $\eta$ and the isomorphism $\alpha(i, p)^{-1}$ is induced by $\kappa$. If the isomorphism $\alpha$ is induced by another homomorphism $\nu$ of $H$ into $K$ in $H / M$, and if $\nu$ induces $\nu(i, p)$ in $H(i, p) / M(i, p)$, then it follows from Theorem 2 of $I .4$ that $\nu(i, p) \alpha(i, p)^{-1}=1$ or $\nu(i, p)=\alpha(i, p)$; and now it is clear how to complete the proof.

Clearly the equations (1) to (3) of I.4 may be restated here without any change. From (1) in particular we can deduce the following interesting inference.

Corollary 1. If $H / M$ is a true representation of the group $G$, then $(M(i, p) \div H(i, p)) / M$ is, for every $i$ and $p$, a characteristic subgroup of $H / M$.

(21) Baer [1, p. 397]. A direct verification of this fact is almost trivial.

(22) These isomorphisms are "natural" in the sense of Eilenberg-MacLane [2]. 
Applying formulas (2) and (3) of I.4 we obtain the following generalizaization of H. Hopf's invariance theorem $\left({ }^{23}\right)$.

Corollary 2. If $H / M$ and $K / N$ are related representations of the same group $G$, then :

(a) $(H(i, p) \cap H(m, p)) M(i, p) / M(i, p)$ and $(K(i, p) \cap K(m, p)) N(i, p) / N(i, p)$ are isomorphic groups;

(b) $(H(i, p) \cap M(m, p)) M(i, p) / M(i, p)$ and $(K(i, p) \cap N(m, p)) N(i, p) / N(i, p)$ are isomorphic groups.

Corollary 1 shows that invariants of the group $G$ may be obtained by considering the subclass of true representations in a given class of related representations of $G$. We shall now select further subclasses distinguished by the fact that certain series are interlocking.

Theorem 2. Suppose that the $p$-M-series of $H$ and the $p$ - $N$-series of $K$ are both interlocking.

(a) If $H / M$ and $K / N$ are related representations of the same group $G$, then (24)

$$
\begin{aligned}
& H / M(i, p) \sim K / N(i, p), \quad H / H(i, p) \sim K / K(i, p), \\
& M(i-1, p) / M(i, p) \sim N(i-1, p) / N(i, p), \\
& \overline{(H ; \overline{M(i, p))}} \sim \overline{(K ; N(i, p))}, \quad \overline{(H ; H(i, p))} \sim \overline{(K ; K(i, p))} .
\end{aligned}
$$

(b) If $H / M$ is a true representation of $G$, then $(M(i, p) \div M(i-1, p)) / M$ is, for positive $i$, a characteristic subgroup of $H / M$.

(c) If the $p$-M-series of $H$ is strictly $\left({ }^{25}\right)$ interlocking, if $\eta$ is a homomorphism of $H$ into $K$ which induces an isomorphism $\alpha$ of $H / M$ upon $K / N$, and if there exists a homomorphism of $K$ into $H$ which induces $\alpha^{-1}$, then $\eta$ is an isomorphism.

Proof. Suppose that $\eta$ is a homomorphism of $H$ into $K$, that $\kappa$ is a homomorphism of $K$ into $H$, that the isomorphism $\alpha$ of $H / M$ upon $K / N$ is induced by $\eta$, that $\alpha^{-1}$ is induced by $\kappa$. Then we deduce from (4) of I.4 that an isomorphism $\phi(i)$ of $M(i-1, p) / M(i, p)$ upon $N(i-1, p) / N(i, p)$ is inducedby $\eta$ (and it may be shown that $\phi(i)$ is the isomorphism induced by $\alpha$ ). It is now an immediate consequence of Lemma 2 of 1.5 that $\eta$ induces an isomorphism of $H / M(i, p)$ upon $K / N(i, p)$ and of

$$
\overline{(H ; M(i, p))} \text { upon } \overline{(K ; N(i, p))} \text {. }
$$

It is a consequence of Theorem 1 (or of Theorem 2 of I.4) that an isomorphism of $H(i, p) / M(i, p)$ upon $K(i, p) / N(i, p)$ is induced by $\eta$. Thus we deduce from the fact that $\eta$ maps $H / M(i, p)$ isomorphically upon $K / N(i, p)$ the desired assertion that an isomorphism of $H / H(i, p)$ upon $K / K(i, p)$ is induced by $\eta$.

(23) Hopf [1]; cp. furthermore Baer [4, §3, Remark 1] and footnote 13.

(24) $A \sim B$ signifies the isomorphy of the groups $A$ and $B$.

(25) That is, if 1 is the cross cut of the subgroups $M(i, p)$. 
Consequently $H(i-1, p) / H(i, p)$ is mapped isomorphically by $\eta$ upon $K(i-1, p) / K(i, p)$; and hence we may deduce from Lemma 2 of 1.5 that

$$
\overline{(H ; H(i, p))} \text { and } \overline{(K ; K(i, p))}
$$

are isomorphic groups. Applying Theorem 3 of $\mathrm{I} .1$ and the lemma of I.4 it follows that $(M(i, p) \div M(i-1, p)) / M$ is mapped by $\alpha$ upon $(N(i, p)$ $\div N(i-1, p)) / N$. We infer from (b) of Lemma 2 of $I .5$ that $\eta$ is an isomorphism if 1 is the cross cut of the groups $M(i, p)$. Now it is clear how to complete the proof.

Note that the isomorphism of $H / M(i, p)$ which is induced by $\eta$ is, in general, not uniquely determined by the isomorphism $\alpha$ of $H / M$ upon $K / N$.

In the course of the proof of (a) we verified the following interesting fact.

Corollary 2. If the $p$-M-series of $H$ and the $p$ - $N$-series of $K$ are both interlocking, and if $H / M$ and $K / N$ are related representations of the same group $G$, then

$$
\overline{(H ; M(i, p))} / \overline{(M ; M(i, p))} \text { and } \overline{(K ; N(i, p))} / \overline{(N ; N(i, p))}
$$

are equivalent representations of $G$.

The following application of the results of this and the preceding section may be worth noting.

THEOREM 3. If $H$ is a splitting extension of its normal subgroup $M$, then $H(i, p) \cap M=M(i, p)$ for every $i$ and $p$.

Proof. Let $K=H / M$ and $N=1$. Then it follows from the theorem of II.1 that $H / M$ and $K / N$ are similar representations of the same group. Hence it follows from (b) of Corollary 2 that $(H(i, p) \cap M) M(i, p) / M(i, p)$ and $(K(i, p) \cap N) N(i, p) / N(i, p)=1$ are isomorphic groups. Consequently $M(i, p)$ $=(H(i, p) \cap M) M(i, p)$ or $H(i, p) \cap M \leqq M(i, p)$. But $M(i, p)$ is always part of both $M$ and $H(i, p)$, proving the desired equality.

It is easy to construct examples which show that the conditions which are by Theorem 3 necessary for splitting certainly do not suffice to assure splitting.

II.3. Comparison of invariant subgroups( $\left.{ }^{26}\right)$. If $H / M$ is a representation of the group $G$, and if $N$ is a normal subgroup of $H$ which is part of $M$, then $(H / N) /(M / N)$ is a representation of $G$ too. This latter representation may be more advantageous than the first one, since a certain $p$ - $M$-series of $H$ may not be interlocking whereas the $p-[M / N]$-series of $H / N$ is interlocking. On the other hand it may happen that $H / M$ is a true representation of $G$, but $(H / N) /(M / N)$ is not. If, however, $N$ is fully invariant relative to $M$, then $(H / N) /(M / N)$ is a true representation of $G$ whenever $H / M$ is a true representation. This condition, though sufficient, is certainly not necessary.

(26) See in this context Baer $[3, \S 1]$. 
Witness representations of groups $G$ all of whose automorphisms are inner.

Suppose now that $H / M$ and $K / N$ are similar representations of the same group $G$, that $M^{*}$ is a subgroup of $M$ which is fully invariant relative to $M$ in $H$, and that $N^{*}$ is a subgroup of $N$ which is fully invariant relative to $N$ in $K$. Then $\left(H / M^{*}\right) /\left(M / M^{*}\right)$ and $\left(K / N^{*}\right) /\left(N / N^{*}\right)$ are certainly both true representations of $G$, but there is no reason to assume that they are similar representations of $G$. Thus there arises the problem of comparing subgroups of different groups. The following concepts will be needed for our discussion of this problem.

If $M$ and $N$ are normal subgroups of the groups $H$ and $K$ respectively, then $(H \rightarrow K ; M \rightarrow N)$ is the set of all the homomorphisms of $H$ into $K$ which map $M$ into part of $N$. In particular $(H \rightarrow K ; 1 \rightarrow 1)=(H \rightarrow K)$ is the set of all the homomorphisms of $H$ into $K$.

If $S$ and $T$ are subsets of the groups $H$ and $K$ respectively, and if $\mathbf{P}$ is a set of homomorphisms of $H$ into $K$, then we denote by $S^{\mathrm{P}}$ the subgroup generated by all the elements $S^{\phi}$ for $s$ in $S$ and $\phi$ in $\mathrm{P}$, and by ${ }^{\mathbf{P}} T$ the subgroup generated by all the elements $x$ in $H$ such that $x^{\phi}$ is in $T$ for every $\phi$ in P.

The subgroup ${ }^{\mathbf{P}} T$ should be well distinguished from the inverse image of $T$ under $\mathrm{P}$ which, in general, will be a larger subgroup. If in particular $T$ happens to be a subgroup of $K$, then ${ }^{\mathbf{P}} T$ consists exactly of those elements $x$ in $H$ whose images under homomorphisms in $\mathrm{P}$ belong to $T$, in symbols: ${ }^{\mathbf{P}} T=\left(x^{\mathbf{P}} \leqq T\right)$.

Theorem 1. Suppose that $M$ and $N$ are normal subgroups of the groups $H$ and $K$ respectively, and that $\mathrm{A}=(H \rightarrow K ; M \rightarrow N)$ and $\mathrm{B}=(K \rightarrow H ; N \rightarrow M)$.

(a) If $S$ is a subgroup of $H$, then $S^{\mathrm{A}}$ and ${ }^{\mathrm{B}} S$ are fully invariant relative to $N$ in $K ; S^{\mathbf{A}}=\left(S^{\mathbf{A}}\right)^{\mathbf{B}},{ }^{\mathbf{A}} S={ }^{\mathrm{A}}\left({ }^{\mathbf{B}} S\right)$; and $\left({ }^{\mathbf{B}} S\right)^{\mathbf{B}} \leqq S \leqq{ }^{\mathbf{A}}\left(S^{\mathbf{A}}\right)$.

(b) If the subgroup $S$ of $H$ is fully invariant relative to $M$, then $S^{\mathrm{A}} \leqq{ }^{\mathrm{B}} S$ and $\left(S^{\mathbf{A}}\right)^{\mathbf{B}} \leqq S \leqq{ }^{\mathbf{A}}\left({ }^{\mathbf{B}} S\right)$.

(c) If $H / M$ and $K / N$ are similar representations of the same group $G$, and if the normal subgroups $S$ and $T$ of $H$ and $K$ respectively satisfy $T^{\mathrm{B}} \leqq S \leqq M$ and $S^{\mathrm{A}} \leqq T \leqq N$, then $(H / S) /(M / S)$ and $(K / T) /(N / T)$ are similar representations of $G$. If in particular $T^{\mathrm{BA}}=T$ and $S^{\mathrm{AB}}=S$, then $T^{\mathrm{B}}=S$ and $S^{\mathrm{A}}=T$.

The proofs of all these facts are fairly immediate and may safely be left to the reader.

In order to obtain more precise results we have to consider pairs of groups $H, K$ which are in a certain sense "locally isomorphic." To define this relation we have to introduce the concept "retract of a group" which is not only of great interest in itself, but will prove of importance throughout our investigations. The subgroup $R$ of the group $G$ may be termed (in analogy to a topological concept $\left({ }^{27}\right)$ ) a retract of $G$, if there exists an idempotent endomorphism $\nu$ of $G$

(27) W. Hurewicz pointed out many years ago, in a conversation with the author, the desirability of translating the concept "retract" from topology to group theory. 
such that $R=G^{\nu}$. If $\nu$ is an idempotent endomorphism of $G, K$ its kernel $\left({ }^{28}\right)$ and $R=G^{\nu}$, then every element in $R$ is a fixed element under $\nu$ so that $\nu$ induces the identity in $G / K$. One and only one element of $R$ is contained in every coset of $G / K$ so that $G$ is a splitting extension of $K$ by $R$; and conversely every splitting extension $G$ of a normal subgroup $K$ by $G / K$ leads to retracts. It should be noted, however, that the kernel $K$ of the idempotent endomorphism $\nu$ need not be a retract of $G$. Two extreme instances of retracts may be mentioned: the free and the direct factors of a group. The latter may be characterized as those retracts which are at the same time normal subgroups.

If $R$ is a retract of $G$, and if $J$ is a fully invariant subgroup of $G$, then $(J R) / J$ is a retract of $G / J$, since every idempotent endomorphism of $G$ with image $R$ induces in $G / J$ an idempotent endomorphism with image $(J R) / J$.

TheOREM 2. (a) The subgroup $R$ of the group $G$ is a retract of $G$ if, and only if, there exists to every homomorphism $\eta$ of $R$ a homomorphism $\gamma$ of $G$ satisfying $G^{r}=R^{r}$ and $x^{\gamma}=x^{\eta}$ for $x$ in $R$.

(b) If $R$ is a retract and $S$ a fully invariant subgroup of $G$, then $S^{(G \rightarrow R)}$ $=S \cap R={ }^{(G \rightarrow R)} S \cap R$.

Proof. If $\nu$ is an idempotent endomorphism of $G$, mapping $G$ upon $R$, then $\gamma=\nu \eta$ for $\eta$ a given homomorphism of $R$ is a homomorphism of $G$ which meets the requirements of (a). If conversely the condition of (a) is satisfied by the subgroup $R$ of $G$, then put $\eta=1$. There exists thèrefore an endomorphism $\nu$ of $G$ which maps $G$ upon $R$ and which leaves every element in $R$ invariant, showing that $R$ is a retract of $G$. This proves (a).

Suppose now that $R$ is a retract of $G, \nu$ an idempotent endomorphism of $G$ which maps $G$ upon $R$, and that $S$ is a fully invariant subgroup of $G$. Then $\nu(R \rightarrow R) \leqq(G \rightarrow R)=(G \rightarrow R) \nu=(G \rightarrow G) \nu$. Since $S$ is a fully invariant subgroup of $G$, we have $S^{(G \rightarrow G)}=S \leqq{ }^{(G \rightarrow R)} S$ and $S^{\nu}=S \cap G^{\nu}=S \cap R$. Now it is clear how to finish the proof of (b).

THEOREM 3. If every element in the group $G$ is contained in a retract of $G$ which is isomorphic to some subgroup of the group $H$, then $M^{(G \rightarrow H)}=1$, for $M a$ not vacuous subset of $G$, implies $M=1$.

Proof. If $x \neq 1$ is an element in $G$, then there exists a retract $R$ of $G$ which contains $x$ and an isomorphism $\alpha$ of $R$ into $H$. It is a consequence of (a) of Theorem 2 that $\alpha$ is induced by a homomorphism of $G$ into $H$ which certainly does not map $x$ upon 1 , proving our contention.

THEOREM 4. If every element in the group $G$ is contained in some retract of $G$ which is isomorphic to a suitable retract of the group $H$, and if $\mathbf{A}=(G \rightarrow H)$ and $\mathbf{B}=(H \rightarrow G)$, then:

(28) The kernel of a homomorphism consists of the elements mapped upon 1 by the homomorphism. 
(i) ${ }^{\mathrm{AB}} S=\left({ }^{\mathrm{B}} S\right)^{\mathrm{B}} \leqq S \leqq{ }^{\mathrm{A}}\left(S^{\mathrm{A}}\right)=S^{\mathrm{AB}}$ for every subgroup $S$ of $G$.

(ii) $J=J^{\mathrm{AB}}={ }^{\mathrm{AB}} J$ for every fully invariant subgroup $J$ of $G$.

(iii) ${ }^{\mathrm{A}} T \leqq T^{\mathrm{B}}$ for every subgroup $T$ of $H$.

(iv) ${ }^{\mathrm{A}} K=K^{\mathrm{B}}$ for every fully invariant subgroup $K$ of $H$.

Proof. If $x$ is an element in the subgroup $S$ of $G$, then there exist isomorphic retracts $U$ and $V$ of the groups $G$ and $H$ respectively such that $x$ is in $U$. One proves readily the existence of a homomorphism $\gamma$ of $G$ into $H$ and of a homomorphism $\eta$ of $H$ into $G$ which induce reciprocal isomorphisms between $U$ and $V$. Clearly $x^{\gamma \eta}=x$ so that $x$ belongs to $S^{\mathrm{AB}}$. Consequently:

(i') $S \leqq S^{\mathrm{AB}}$ for $S$ a subgroup of $G$.

If $T$ is a subgroup of $H$, then $\left({ }^{\mathrm{A}} T\right)^{\mathrm{A}} \leqq T$ by (a) of Theorem 1 , and hence it follows from (i') that ${ }^{\mathrm{A}} T \leqq\left({ }^{\mathrm{A}} T\right)^{\mathrm{AB}}=\left(\left({ }^{\mathrm{A}} T\right)^{\mathrm{A}}\right)^{\mathrm{B}} \leqq T^{\mathrm{B}}$, proving (iii). If $T$ happens to be a fully invariant subgroup of $H$, then we infer $T^{B} \leqq{ }^{A} T$ from (b) of Theorem 1 , and thus (iv) is a consequence of (iii).

If $S$ is a subgroup of $G$, then $S^{\mathrm{A}}$ and ${ }^{\mathrm{B}} S$ are fully invariant subgroups of $H$. Substituting $S^{\mathbf{A}}$ and ${ }^{\mathbf{B}} S$ for $K$ in (iv) we obtain therefore ${ }^{\mathrm{A}}\left(S^{\AA}\right)=S^{\mathrm{AB}}$ and ${ }^{\mathrm{B}} S=\left({ }^{\mathrm{B}} S\right)^{\mathrm{B}}$, and (i) is an immediate consequence of (a) of Theorem 1. Likewise (ii) is an immediate consequence of (i) and (b) of Theorem 1.

CoROLlary 1. If every element in the group $G$ is contained in a retract of $G$ which is isomorphic to a retract of the group $H$, and if the subgroups $M$ and $N$ of $G$ and $H$ respectively satisfy $M^{(G \rightarrow H)} \leqq N$ and $N^{(H \rightarrow G)} \leqq M$, then $M$ is a fully invariant subgroup of $G$ and

$$
M=N^{(H \rightarrow G)}=(G \rightarrow B) N .
$$

Proof. It is an immediate consequence of (i) of Theorem 4 that $M \leqq M^{(G \rightarrow H)(H \rightarrow G)} \leqq N^{(H \rightarrow G)} \leqq M$ or $M=N^{(H \rightarrow G)}$. From $M^{(G \rightarrow H)} \leqq N$ we deduce $M \leqq{ }^{(G \rightarrow H)} N$ and from (iii) of Theorem 4 we deduce ${ }^{(G \rightarrow H)} N \leqq N^{(H \rightarrow G)} \leqq M$, completing the proof.

Remark 1. If $N$ happens to be a fully invariant subgroup of the group $H$, then we deduce from (iv) of Theorem 4 that $M=N^{(B \rightarrow G)}$ and $M={ }^{(G \rightarrow B)} N$ are equivalent properties of the subgroup $M$ of $G$, and from $M=N^{(H \rightarrow G)}$ we deduce $M^{(G \rightarrow H)}=N^{(H \rightarrow G)(G \rightarrow H)} \leqq H$, showing that in case $N$ is fully invariant the two equations are not only necessary, but even sufficient for the validity of the two inequalities.

If $G$ is any group, then it will be convenient to denote by $J(G)$ the partially ordered set of all the fully invariant subgroups of G. Projectivities (or isomorphisms) of partially ordered sets are 1:1 and monotone increasing maps. Using this terminology we obtain a significant interpretation of Theorem 4.

If every element in the group $G$ is contained in some retract of $G$ which is isomorphic to a suitable retract of the group $H$, then projectivities of $J(G)$ into part of $J(H)$ are obtained both by mapping $X$ in $J(G)$ upon $X^{(G \rightarrow H)}$ and by mapping $X$ in $J(G)$ upon ${ }^{(H \rightarrow G)} X$. These two projectivities need not be equal. 
If, however, in addition to the hypothesis already made every element in $H$ is contained in some retract of $H$ which is isomorphic to a suitable retract of $G$, then these two projectivities are equal and map $J(G)$ upon the whole system $J(H)$ so that $J(G)$ and $J(H)$ are essentially the same.

THEOREM 5. If $R$ and $S$ are isomorphic retracts of the groups $H$ and $K$ respectively, and if the normal subgroups $F$ and $V$ of $H$ and $K$ respectively satisfy $F^{(H \rightarrow K)} \leqq V$ and $V^{(K \rightarrow H)} \leqq F$, then $(R F) / F$ and $(S V) / V$ are isomorphic retracts of $H / F$ and $K / V$ respectively.

Proof. It has been pointed out before that $(R F) / F$ is a retract of $H / F$ and that $(S V) / V$ is a retract of $K / V$. There exists an isomorphism $\phi$ of $R$ upon $S$. It is a consequence of (a) of Theorem 2 that homomorphisms of retracts are induced by homomorphisms of the group. Hence $(R \cap F)^{\phi} \leqq R^{\phi}$ $\cap F^{(B \rightarrow K)} \leqq S \cap V$ and likewise $(S \cap V)^{\phi^{-1}} \leqq R \cap F$ or $S \cap V \leqq(R \cap F)^{\phi}$. Consequently we have $S \cap V=(R \cap F)^{\phi}$, proving that $\phi$ induces an isomorphism of $R /(R \cap F)$ upon $S /(S \cap V)$. But these groups are isomorphic to $(R F) / F$ and $(S V) / V$ respectively, completing the proof.

CoRollary 2. If $M$ and $N$ are normal subgroups of the groups $G$ and $H$ respectively such that $M^{(G \rightarrow H)} \leqq N$ and $N^{(H \rightarrow G)} \leqq M$, and if every element in $G$ is contained in some retract of $G$ which is isomorphic to a suitable retract of $H$, then every element in $G / M$ is contained in some retract of $G / M$ which is isomorphic to a suitable retract of $H / N$.

This is an almost immediate consequence of Theorem 5 .

(c) of Theorem 1 and Corollaries 1 and 2 show the importance of the relation $M^{(\theta \rightarrow H)} \leqq N$ for $M$ and $N$ normal subgroups of the groups $G$ and $H$ respectively. It is, therefore, desirable to find criteria for this inequality.

THeOREM 6. Suppose that $M$ and $N$ are normal subgroups of the groups $H$ and $K$ respectively, and that there exists a homomorphism $\eta$ of $H$ into $K$ which induces an isomorphism of $H / M$ into $K / N$.

(a) If $L$ is a subgroup of $H$ such that $L^{(H \rightarrow K)} \leqq N$, then $L \leqq M$.

(b) If the fully invariant subgroup $J$ of $K$ is part of $N$, then $J^{(K \rightarrow H)} \leqq M$.

Proof. If $x$ is in $L$, and if $L^{(H \rightarrow K)} \leqq N$, then $x^{\eta}$ belongs to $N$. Hence $(M x)^{\eta} \leqq N$. But $\eta$ induces an isomorphism of $H / M$ into $K / N$. Consequently $M x=M$ so that $x$ is in $M$ or $L \leqq M$, proving (a).

If the fully invariant subgroup $J$ of $K$ is part of $N$, then $J^{(K \rightarrow H)}$ is a subgroup of $H$ satisfying: $\left[J^{(K \rightarrow H)}\right]^{(H \rightarrow K)} \leqq J^{(K \rightarrow K)}=J \leqq N$, and we deduce from (a) that $J^{(K \rightarrow H)} \leqq M$.

Corollary 3. Suppose that $M$ and $N$ are normal subgroups of the groups $H$ and $K$ respectively, and that there exists a homomorphism of $H$ into $K$ which 
induces an isomorphism of $H / M$ into $K / N$ and a homomorphism of $K$ into $H$ which induces an isomorphism of $K / N$ into $H / M$.

(a) If $J$ is a fully invariant subgroup of $H$, then the inequalities $J \leqq M$ and $J^{(\boldsymbol{H} \rightarrow K)} \leqq N$ imply each other.

(b) If $U$ and $V$ are greatest fully invariant subgroups of the groups $H$ and $K$ respectively with the properties $U \leqq M$ and $V \leqq N$, then

$$
U^{(\boldsymbol{H} \rightarrow K)} \leqq V \text { and } V^{(\boldsymbol{K} \rightarrow \boldsymbol{B})} \leqq U .
$$

Proof. If $J$ is a fully invariant subgroup of $H$, and $J \leqq M$, then $J^{(H \rightarrow K)} \leqq N$ by (b) of Theorem 6 , and if conversely $J^{(B \rightarrow K)} \leqq N$, then $J \leqq M$ by (a) of Theorem 6.

If $U$ is a fully invariant subgroup of $H$ and $U \leqq M$, then $U^{(H \rightarrow K)}$ is a fully invariant subgroup of $K$ which is, by (b) of Theorem 6, part of $N$. If $V$ is a greatest fully invariant subgroup of $K$ which is part of $N$, then these facts imply $U^{(B \rightarrow K)} \leqq V$. The second inequality of (b) is verified in a like manner.

Remark 2. The general hypotheses of Corollary 3 are satisfied, in particular, whenever $H / M$ and $K / N$ are related representations of the same group $G$.

Remark 3. The join of all the fully invariant subgroups of the group $H$ which are part of the subgroup $M$ of $H$ is readily seen to be a fully invariant subgroup too so that there exists one and only one greatest fully invariant subgroup of $H$ which is part of $M$.

The results of Theorem 6 and Corollary 3 should be compared with Corollary 1 and (c) of Theorem 1.

II.4. Similar groups. The groups $H$ and $K$ shall be termed similar, if there exist, to every pair of homomorphisms $\eta$ and $\kappa$ of $H$ and $K$ respectively which satisfy $H^{n}=K^{\star}$, a homomorphism $\eta^{\prime}$ of $H$ into $K$ and a homomorphism $\kappa^{\prime}$ of $K$ into $H$ such that $\eta=\eta^{\prime} \kappa$ and $\kappa=\kappa^{\prime} \eta$.

The symmetry of this similarity relation is obvious. But there exist groups which are not self-similar, and likewise it is impossible to prove transitivity of this concept of similarity, since every group is similar to the identity group, though there exist dissimilar groups. On the other hand there exist trivial cases of similarity; for the groups $H$ and $K$ would be certainly similar, if they do not possess any isomorphic quotient groups excepting 1.

THEOREM 1. The following condition is necessary and sufficient for the two groups $H$ and $K$ to be similar.

If $H / M$ and $K / N$ are representations of the same group $G$, then they are similar representations of $G$.

Proof. If $H$ and $K$ are similar, and if $H / M$ and $K / N$ are representations of the same group $G$, then let $\alpha$ be an isomorphism of $H / M$ upon $K / N$. A homomorphism $\eta$ of $H$ upon $K / N$ is defined by mapping the element $x$ in $H$ upon the coset $(M x)^{\alpha}$ of $K / N$, and a homomorphism $\kappa$ of $K$ upon $K / N$ is defined by mapping the element $y$ in $K$ upon the coset $N y$. Clearly $H^{\eta}=K^{\star}(=K / N)$. Hence there exists a homomorphism $\eta^{\prime}$ of $H$ into $K$ such 
that $\eta=\eta^{\prime} \kappa$. If $X$ is a coset of $H / M$, then $N X^{\eta^{\prime}}=X^{\eta^{\prime} \kappa}=X^{\eta}=X^{\alpha}$, showing that $\alpha$ is induced by $\eta^{\prime}$. Likewise one shows the existence of a homomorphism of $K$ into $H$ which induces $\alpha^{-1}$. Thus $H / M$ and $K / N$ are similar representations of $G$.

Assume conversely that the condition is satisfied by $H$ and $K$ and that $H^{\eta}=K^{*}$ is satisfied by the homomorphisms $\eta$ and $\kappa$ of $H$ and $K$ respectively. Denote by $M$ the kernel of $\eta$ and by $N$ the kernel of $\kappa$. Then $H / M$ and $K / N$ are isomorphic groups, since they are both isomorphic to $G=H^{*}=K^{*}$. As a matter of fact $\eta$ induces an isomorphism $\eta^{*}$ of $H / M$ upon $G$ and $\kappa$ induces an isomorphism $\kappa^{*}$ of $K / N$ upon $G$, and there exists therefore one and only one isomorphism $\alpha$ of $H / M$ upon $K / N$ such that $\eta^{*}=\alpha \kappa^{*}$. But $H / M$ and $K / N$ are by hypothesis similar representations of the group $G$, and hence there exists a homomorphism $\eta^{\prime}$ of $H$ into $K$ which induces $\alpha$ in $H / M$. If $x$ is an

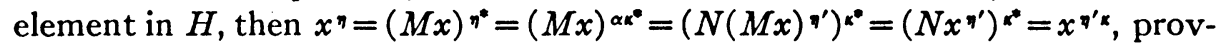
ing $\eta=\eta^{\prime} \kappa$, and likewise one shows the existence of a homomorphism $\kappa^{\prime}$ of $K$ into $H$ such that $\kappa=\kappa^{\prime} \eta$ so that $H$ and $K$ have been shown to be similar groups.

CoRollary 1. If $S$ and $T$ are normal subgroups of the groups $H$ and $K$ respectively, and if $S^{(H \rightarrow K)} \leqq T$ and $T^{(K \rightarrow H)} \leqq S$, then the similarity of $H / S$ and $K / T$ is a consequence of the similarity of $H$ and $K$.

This is an immediate consequence of Theorem 1 and (c) of Theorem 1 of II.3.

Remark. Exactly as we distinguished related and similar representations, so one may differentiate between related and similar groups. The former would be defined as follows: The groups $H$.and $K$ are related groups, if they meet the following requirement.

If $M$ and $N$ are normal subgroups of $H$ and $K$ respectively such that $H / M \sim K / N$, then there exist homomorphisms of $H$ into $K$ and of $K$ into $H$ which induce reciprocal isomorphisms between $H / M$ and $K / N$.

Though this concept is quite natural and seems to be useful in applications, we shall not discuss it in this first part of our investigation.

CoRollary 2. If the groups $H$ and $K$ are similar, if the groups $K$ and $L$ are similar, and if every homomorphic image of both groups $H$ and $L$ is a homomorphic image of $K$ too, then $H$ and $L$ are similar.

Remark. The impossibility of omitting the hypothesis concerning the existence of sufficiently many homomorphic images of $K$ is a consequence of the fact, pointed out before, that similarity is, in general, not transitive.

Proof. Suppose that $H / M$ and $L / N$ are representations of the same group $G$. Then we infer from our hypothesis the existence of a normal subgroup $S$ of $K$ such that $K / S$ is a representation of the group $G$ too. It is a consequence of the similarity of $H$ and $K$ and of Theorem 1 that $H / M$ and $K / S$ are similar 
representations of $G$, and likewise one sees that $K / S$ and $L / N$ are similar representations of $G$. But similarity of representations is transitive so that $H / M$ and $L / N$ are similar representations of $G$. Now the similarity of the groups $H$ and $L$ is an immediate consequence of Theorem 1.

THEOREM 2. The group $G$ is isomorphic to a retract of the self-similar group $H$ if, and only if, (i) $G$ is a homomorphic image of $H$, and (ii) $G$ and $H$ are similar groups.

Proof. If conditions (i) and (ii) are satisfied, then we infer from the theorem of II.1 that every representation of $G$ in the form $H / N$ for $N$ a normal subgroup of $H$ is a splitting extension of $N$ by $G$, showing the sufficiency of the conditions. If, conversely, $G$ is a retract of $H$, then there exists an idempotent endomorphism $\epsilon$ of $H$ such that $G=H^{e}$. Suppose now that $M$ and $K$ are normal subgroups of $G$ and $H$ respectively and that $\alpha$ is an isomorphism of $G / M$ upon $H / K$. If $E$ is the kernel of the endomorphism $\epsilon$, then $G / M$ and $H / M E$ are essentially the same, since $H=G E$ and $1=G \cap E, M=G \cap M E$. Thus an isomorphism $\beta$ of $H / M E$ upon $H / K$ is defined by mapping the coset $X$ of $H / M E$ upon the coset $(X \cap G)^{\alpha}=X^{\beta}$ of $H / K$. Since $H$ is self-similar, there exists an endomorphism $\eta$ of $H$ inducing $\beta$ in $H / M E$ and an endomorphism $\kappa$ of $H$ inducing $\beta^{-1}$ in $H / K$. But $\eta$ induces a homomorphism $\gamma$ of $G$ into $H$; and it is clear that $\alpha$ is induced by $\gamma$ in $G / M$. Furthermore $\kappa \epsilon$ is a homomorphism of $H$ into $G$ which induces $\alpha^{-1}$ in $H / K$. Thus $H / K$ and $G / M$ have been shown to be similar representations of the same group, and the similarity of $G$ and $H$ is a consequence of Theorem 1 .

COROllaRY 3. Retracts of similar self-similar groups are similar.

Proof. Suppose that the groups $R$ and $S$ are retracts of the self-similar groups $H$ and $K$ respectively and that $H$ and $K$ are similar groups. It is a consequence of Theorem 2 that $R$ and $H$ are similar groups. If $G$ is a homomorphic image of both $R$ and $K$, then $G$ is a homomorphic image of $H$, since $R$ is a homomorphic image of $H$. Thus it follows from Corollary 2 that $R$ and $K$ are similar groups. It is a consequence of Theorem 2 that $K$ and $S$ are similar groups. If the group $L$ is a homomorphic image of both $R$ and $S$, then $L$ is a homomorphic image of $K$, since $L$ is a homomorphic image of $S$. Thus the similarity of $R$ and $S$ is a consequence of Corollary 2, as we intended to show.

If $\Xi$ is any class of groups, then denote by $\Xi_{r}$ the class of all the retracts of groups in $\Xi$ and by $\Xi_{h}$ the class of all the homomorphic images of groups in $\Xi$. Clearly $\Xi \leqq \Xi_{r} \leqq \Xi_{h}$ and $\Xi_{r}=\Xi_{r r}, \Xi_{h}=\Xi_{r h}=\Xi_{h h}$.

THEOREM 3. If $\Xi$ is a class of similar groups $\left({ }^{29}\right)$, then the following properties of a group $G$ in $\Xi_{h}$ are equivalent:

(29) That is, any two groups in $\Xi$ are similar and every group in $\Xi$ is self-similar. 
(i) $G$ belongs to $\Xi_{r}$.

(ii) $G$ is similar to every group in $\Xi_{r}$.

(iii) $G$ is similar to every group in $\Xi$.

Proof. It is a consequence of Corollary 3 that (i) implies (ii), and it is obvious that (iii) is a consequence of (ii). That (i) is implied by (iii) may be inferred from Theorem 2 .

CoRollary 4. If $\Xi$ is a class of similar groups, and if $G$ is a group in $\Xi_{h}$, then there exists one and only one class of similar representations of the group $G$ in the form $F / N$ for $F$ in $\Xi$.

This is an immediate consequence of Theorem 1 .

THEOREM 4. The following properties of the fully invariant subgroups $J$ and $K$ of the self-similar group $G$ are equivalent:

(i) $G / J$ and $G / K$ are similar groups.

(ii) $G /(J \cap K)$ is a splitting extension of $(J K) /(J \cap K)$.

(iii) $G / J$ and $G / K$ are isomorphic to retracts of $G /(J \cap K)$.

Proof. We note that $J K$ and $D=J \cap K$ are fully invariant subgroups of $G$, that $J K / D$ is the direct product of $J / D$ and $K / D$ and that these latter groups are normal subgroups of $G / D$, since inner automorphisms of $G / D$ are induced by inner automorphisms of $G$. Furthermore $G / D$ is self-similar, since $D$ is a fully invariant subgroup of the self-similar group $G$ (Corollary 1).

If $G / J$ and $G / K$ are similar groups, then we infer from the self-similarity of $G$ the existence of endomorphisms $\epsilon$ and $\phi$ of $G$ which both induce the identity in $G /(J K)$ and satisfy $J^{\epsilon} \leqq K, K^{\phi} \leqq J$, since $G /(J K)$ is a common quotient group of $G / J$ and $G / K$. But $J$ and $K$ are fully invariant subgroups of $G$ so that $J^{\epsilon} \leqq J \cap K$ and $K^{\phi} \leqq J \cap K$. Clearly $\epsilon \phi$ is an endomorphism of $G$ which induces the identity in $G /(J K)$ and which satisfies

$$
(J K)^{\epsilon \phi} \leqq(J \cap K)^{\phi} K^{\phi} \leqq J \cap K=D .
$$

To every element $x$ in $G$ there exists therefore an element $x^{\prime}$ in $J K$ such that $x^{\epsilon \phi}=x x^{\prime}$, and $x^{\prime \epsilon \phi}$ belongs to $D$. Thus $x^{(\epsilon \phi)^{2}}=\left(x x^{\prime}\right)^{\epsilon \phi}=x^{\epsilon \phi} x^{\prime \epsilon \phi} \equiv x^{\epsilon \phi}$ modulo $D$, proving that $\epsilon \phi$ induces in $G / D$ an idempotent endomorphism with kernel $(J K) / D$. Thus we have shown that (ii) is a consequence of (i).

That (ii) implies (iii) is a consequence of the facts mentioned before that $J / D$ and $K / D$ are normal subgroups of $G / D$ and that $(J K) / D$ is the direct product of $J / D$ and $K / D$.

It is finally a consequence of Corollary 3 that (i) is implied by (iii), since we mentioned before that $G / D$ is self-similar.

Chapter III. Group theoretical properties and Classes OF REPRESENTATIONS

It is well known that every group may be represented as a quotient group 
of a free group. If the group $G$ under consideration is known to belong to some special class of groups, then $G$ may be represented as a quotient group of a "reduced free group"; for example, if $G$ satisfies ${ }^{i} G=1$ or $G^{(i)}=1$ or $G^{i}=1$, then $G$ may be represented as a quotient group of some group $F /{ }^{i} F$ or $F / F^{(i)}$ or $F / F^{i}$ for $F$ a free group, and these latter groups are reduced free groups. It is the object of this chapter to discuss the classes of representations arising in this fashion.

III.1. Free groups. A free group $F$ is determined by one invariant only which we call the rank $r(F)$ of $F$. The rank of a free group is the number of elements in every free set of generators. If $F$ is a free group, then $F /(F, F)$ is usually termed a free abelian group; and $r(F)$ is at the same time the rank of the free abelian group $F /(F, F)$ (in the terminology customary in the theory of free abelian groups).

Every subgroup of a free group is a free group (Schreier's theorem), and the free group of rank 2 contains a free group of countably infinite rank $\left({ }^{30}\right)$, for example, its commutator subgroup.

LEMMA 1. Every homomorphism of the free group $F$ into the quotient group $G / N$ is induced by a homomorphism of $F$ into $G$.

Proof. Denote by $\eta$ some homomorphism of $F$ into $G / N$ and by $B$ a free set of generators of $F$. Select in the coset $b \eta$, for $b$ in $B$, an element $r(b)$ so that $b^{\eta}=N r(b)$ for every $b$ in $B$. Since $B$ is a free set of generators of the free group $F$, there exists one and only one homomorphism $\phi$ of $F$ into $G$, satisfying $b^{*}=r(b)$ for $b$ in $B$. Denote finally by $\nu$ the homomorphism of $G$ upon $G / N$ which maps every element in $G$ upon its coset modulo $N$. It is clear then that $\phi \nu=\eta$, showing that $\eta$ is induced by $\phi$.

Remark 1. If $\eta$ is a homomorphism of the quotient group $G / N$ into the group $H$, then $\eta$ is induced by the homomorphism $\nu \eta$ of $G$ into $H$ where $\nu$ is the natural homomorphism of $G$ upon $G / N$.

THEOREM 1. The group $G$ is a free group if, and only if, it is similar to every free group.

Proof. If $M$ and $N$ are normal subgroups of the free groups $F$ and $V$ respectively, then it follows from Lemma 1 (and Remark 1) that every homomorphism of $F / M$ into $V / N$ is induced by a homomorphism of $F$ into $V$, and that every homomorphism of $V / N$ into $F / M$ is induced by a homomorphism of $V$ into $F$. This implies in particular that $F / M$ and $V / N$ are similar representations of the group $S$ if they are both representations of $S$. Thus we deduce from Theorem 1 of II.4 the similarity of $F$ and $V$, proving the necessity of our condition.

Assume now that the group $G$ is similar to every free group. There exists a representation $F / N$ of $G$ as a quotient group of a free group $F$. Thus the

(30) Levi [1]. 
free group $F$ and its quotient group $F / N$ are similar, and we infer from Theorem 1 of II.4 that $F / N$ and the trivial representation $G / 1$ of $G$ are similar representations of $G$. Hence it follows from the theorem of II.1 that $F$ is a splitting extension of $N$ by $G$. Consequently $G$ is isomorphic to a subgroup of the free group $F$. Since subgroups of free groups are free too, we have shown that $G$ is free, and this completes the proof.

Remark 2. It is apparent from the second part of the proof that it suffices to assume the similarity of $G$ and of a free group of sufficiently large rank. On the other hand it does not suffice to assume the similarity of $G$ and of some free group, since every group is similar to the free group 1.

The following fact has already been verified in the first part of the proof of Theorem 1 ; it is clearly an immediate consequence of Theorem 1 and Theorem 1 of II.4, and shall be restated because of its importance.

Corollary 1. Any two representations of the group $G$ as quotient groups of free groups are similar.

We say that the representation $H / M$ of the group $G$ is not redundant $\left.{ }^{(31}\right)$ if there exists a set of representatives of the cosets, not $M$, of $H / M$ which generates the group $H$. Clearly many representations are redundant. Note furthermore that a representation may be not redundant, though some of the sets of representatives generate proper subgroups.

The most important instance of a not redundant representation is the regular representation. The representation $H / M$ of the group $G$ is said to be regular if there exists a set of representatives of the cosets, not $M$, of $H / M$ which is at the same time a free set of generators of $H$ (so that $H$ is in particular a free group).

The existence of a regular representation of the group $G$ may be seen as follows. Let $F$ be a free group such that $r(F)+1$ is the finite or infinite number of elements in $G$. If $B$ is any free set of generators of $F$, then there exists a $1: 1$ correspondence $b(g)$ mapping the set of elements $g \neq 1$ upon the whole set $B$. It will be convenient to put $b(1)=1$. Since $F$ is a free group and $B$ a free set of generators of $F$, there exists one and only one homomorphism $\eta$ of $F$ upon $G$ which maps $b(g)$ upon $g$. The kernel of this homomorphism is readily $\left({ }^{32}\right)$ seen to be the smallest normal subgroup $N$ of $F$ which contains all the elements

$$
b(x) b(y) b(x y)^{-1} \quad \text { for } x, y \text { in } G .
$$

It is fairly obvious that every regular representation of $G$ has this form, and that therefore any two regular representations of the group $G$ are equivalent, a fact that we may obtain too as a special case of a theorem proved below. Thus the regular representation is a normal form for representations of the

(3i) Much use has been made of this and the following concepts in Baer [4].

(32) This fact is well known; see Magnus [2, p. 14]. 
group $G$, and every invariant of $F$ relative to $N$, for $F / N$ the regular representation of $G$, is an absolute invariant of the group $G$.

The universal character of the regular representation is expressed by the following statement.

THEOREM 2. If $F / N$ is the regular representation of the group $G$, if the representation $H / M$ of the group $G^{*}$ is not redundant, and if $\gamma$ is a homomorphism of $F / N$ upon $H / M$, then there exists a homomorphism $\eta$ of $F$ upon $H$ with the following properties:

(i) $\eta$ induces the homomorphism $\gamma$ of $F / N$ upon $H / M$.

(ii) $F^{\eta}=H$ and $N^{\eta}=M$.

(iii) $F(i, p)^{\eta}=H(i, p)$ and $N(i, p)^{\eta}=M(i, p)$ for every $i$ and $p\left({ }^{(33}\right)$.

Proof. Since $F / N$ is the regular representation of the group $G$, there exists a set of representatives $b(g)$ of the cosets of $F / N$ (such that $N b(g)$ is the coset corresponding to the element $g$ in $G$, and) such that $b(1)=1$ whereas the elements $b(g)$ for $g \neq 1$ form a free set of generators of $F$. Since $H / M$ is a not redundant representation of $G^{*}$, there exists a set of representatives $r(x)$ of the cosets of $H / M$ such that $r(1)=1$ and such that $H$ is generated by the $r(x)$. Since the $b(g)$ for $g \neq 1$ form a free set of generators, there exists one and only one homomorphism $\eta$ of $F$ into $H$ such that:

(iv) $b(x)^{\eta}=r\left(x^{\gamma}\right)$ for $x$ in $F / N$.

Clearly $F^{v}=H$. If $x, y$ are elements in $F / N$, then

$$
\left(b(x) b(y) b(x y)^{-1}\right)^{\eta}=r\left(x^{\gamma}\right) r\left(y^{\gamma}\right) r\left((x y)^{\gamma}\right)^{-1}=r\left(x^{\gamma}\right) r\left(y^{\gamma}\right) r\left(x^{\gamma} y^{\gamma}\right)^{-1},
$$

proving $N^{\eta} \leqq M$, since $N$ is the smallest normal subgroup of $F$ which contains all the elements $b(x) b(y) b(x y)^{-1}$; and now it is evident that $\eta$ induces the homomorphism $\gamma$ of $F / N$ upon $H / M$. If $z$ is an element in $M$, then there exists an element $y$ in $F$ such that $y^{\eta}=z$. Hence $(N y)^{\gamma}=M(N y)^{\eta}=M z=M$. There exists furthermore one and only one element $w$ in $N$ such that $y=w b(N y)$, and we find:

$$
w^{\eta}=w^{\eta} r(M)=w^{\eta} r\left((N y)^{\gamma}\right)=w^{\eta} b(N y)^{n}=(w b(N y))^{\eta}=y^{\eta}=z,
$$

proving $N^{\eta}=M$. Thus we have shown that $\eta$ meets the requirements (i) and (ii). This completes the proof of our theorem, since the equivalence of the properties (ii) and (iii) is easily verified.

We note for future reference the degree of freedom in the construction of the homomorphisms $\eta$ which expresses itself in equation (iv).

The equations (iii) of Theorem 2 have several important and obvious consequences. We mention that $\eta$ induces a homomorphism of $F / F(i, p)$ upon $H / H(i, p)$, of $F / N(i, p)$ upon $H / M(i, p)$ and of $F(i, p) / N(i, p)$ upon $H(i, p) / M(i, p)$. Furthermore $N(i, p) \div F(i, p)$ is mapped by $\eta$ upon $M(i, p)$ $\div H(i, p)$.

(33) For the notations see $\S I .2$. 
III.2. The fully invariant subgroups of free groups. The following theorems concerning fully invariant subgroups of free groups are due to $\mathrm{F}$. Levi:

(a) $\left.{ }^{(34}\right)$ If the fully invariant subgroup $J$ of the free group $F$ is not part of the commutator subgroup $(F, F)$ of $F$, then $F^{n} \leqq J$ for some positive integer $n$.

(b) $\left.{ }^{35}\right)$ If the commutator subgroup $(F, F)$ of the free group $F$ is part of the characteristic subgroup $C$ of $F$, then $C=(F, F) F^{m}$ for some integer $m$.

For our purposes we shall need the following extension of these theorems.

THEOREM 1. Suppose that $J$ is a fully invariant subgroup of the free group $F \neq 1$.

(i) $\left.{ }^{36}\right)$ There exists one and only one integer $n \geqq 0$ such that $F^{n} \leqq J \leqq(F, F) J$ $=(F, F) F^{n}$.

(ii) If $0 \leqq m$, then the inequalities $F^{m} \leqq J$ and $F^{m} \leqq(F, F) J$ imply each other.

(iii) If $0 \leqq n$, then the equations $(F, F) J=(F, F) F^{n}$ and $J=((F, F) \cap J) F^{n}$ imply each other.

Proof. Clearly $(F, F) J$ is a fully invariant subgroup of $F$. Hence there exists by (b) one and only one integer $n \geqq 0$ such that $(F, F) J=(F, F) F^{n}$.

Suppose next that $F^{m} \leqq(F, F) J$ for some integer $m$. There exists a (not vacuous) free set $B$ of generators of $F$. If $b$ is an element in $B$, then $b^{m}$ belongs to $(F, F) J$; and there exist therefore elements $f$ and $j$ in $(F, F)$ and $J$ respectively such that $b^{m}=f j$. There exists furthermore one and only one endomorphism $\eta$ of $F$ which maps $b$ upon itself and every element, not $b$, in $B$ upon 1 . Since $F^{\eta}$ is an infinite cyclic group, we have $(F, F)^{\eta}=1$. Since $J$ is fully invariant, $J^{\eta} \leqq J$ so that $j^{\eta}$ belongs to $J$. But $b^{m}=\left(b^{m}\right)^{\eta}=(f j)^{\eta}=f^{\eta} j^{\eta}=j^{\eta}$, showing that $b^{m}$ belongs to $J$. If $x$ is any element in $F$, then there exists an endomorphism $\epsilon$ of $F$ which maps $b$ upon $x$. Since $J$ is fully invariant, $x^{m}=\left(b^{m}\right)^{\epsilon}$ belongs to $J$ too, and thus we have shown that $F^{m} \leqq J$ is a consequence of $F^{m} \leqq(F, F) J$. This proves (ii), and (i) is an immediate consequence of (ii) and of the results stated in the first paragraph of the proof.

Suppose finally that $(F, F) J=(F, F) F^{n}$ for some $n$. Then every element $j$ in $J$ has the form $j^{\prime} j^{\prime \prime}$ for $j^{\prime}$ in $F^{n}$ and $j^{\prime \prime}$ in $(F, F)$. It is a consequence of (ii) that $F^{n} \leqq J$, proving that $j^{\prime}$ and therefore $j^{\prime \prime}=j^{\prime-1} j$ belong to $J$. Consequently $j^{\prime \prime}$ belongs to $J \cap(F, F)$; and hence we have shown that $J \leqq F^{n}(J \cap(F, F))$. But $F^{n} \leqq J$ and hence $J=((F, F) \cap J) F^{n}$. If conversely $J=((F, F) \cap J) F^{n}$, then $(F, F) J=(F, F) F^{n}$, completing the proof.

The following fact is an immediate consequence of this theorem.

Corollary 1. $F$ is the only fully invariant subgroup $J$ of the free group $F$ which satisfies $(F, F) J=F$.

To apply the results of II.3 on free groups we need the following facts.

(34) Levi [2, p. 93, Satz 3].

(35) Levi [2, p. 91, Sat7 2].

(36) For a similar statement see Neumann [1, p. 523, Theorem 19.1]. 
THEOREM 2. (a) The group $G$ is isomorphic to a retract of the free group $F$ if, and only if, $G$ is a free group of rank not exceeding $r(F)$.

(b) Every element in the not abelian free group $F$ is contained in a retract of $F$ which is isomorphic to a subgroup of the free group $V$ if, and only if, $V$ is not abelian.

(c) Every element in the not abelian free group $F$ is contained in a retract of $F$ which is isomorphic to a retract of the free group $V$ if, and only if, $r(V)<r(F)$ implies the infinity of $r(V)$.

Proof. A retract of a free group is at the same time a subgroup and a homomorphic image of this free group. The first property implies that retracts of free groups are themselves free groups, and the second property shows that the rank of a retract cannot exceed the rank of the original group. This shows the necessity of the conditions of (a). If conversely $G$ is a free group such that $r(G) \leqq r(F)$, and if $B$ is a free set of generators of $F$, then $B$ contains a subset $S$ containing exactly $r(G)$ elements. It is readily seen that the subgroup, generated by $S$, is a retract of $F$ which is isomorphic to $G$, proving the sufficiency of our conditions.

The statements (b) and (c) are readily deduced from (a) and the following two facts : every not abelian free group contains a free subgroup of countably infinite rank, and every element in a free group is contained in a retract of a finite rank.

Remark 1. It is readily seen that every free factor of a (free) group $F$ is a retract of $F$. Whether or not every retract of a free group is a free factor has not yet been decided. It follows, however, from a proof of F. Levi( ${ }^{(37)}$ that every retract of a free group of finite rank is a free factor.

CoRollary 2. If $F$ and $V$ are free groups such that $1 \leqq r(F) \leqq r(V)$, then there exists a projectivity of the partially ordered set of all the fully invariant subgroups of $F$ into the partially ordered set of all the fully invariant subgroups of $V$, and infinity of $r(F)$ implies that these partially ordered sets are projections of each other.

It is an immediate consequence of Theorem 2 and of Theorem 4 of II.3 that $J^{(F \rightarrow V)}$ for $J$ a fully invariant subgroup of $F$ effects the desired projectivity. If $r(F)=1$, then the validity of our statement is obvious.

Remark 2. If $F$ and $V$ are not isomorphic free groups, both of finite rank, then their fully invariant subgroups form essentially different partially ordered sets. This may be illustrated by some examples. The most obvious example is obtained by assuming that $F$ is an infinite cyclic group, $V$ a not abelian free group. Then the only fully invariant subgroups of $F$ are the subgroups $F^{n}$ for $n$ any not negative integer, but $V$ possesses apart from the fully invariant subgroups $V^{n}$ among others the following ones: ${ }^{n} V, V^{(n)}$, and so on. The following example, however, is less trivial.

(37) Levi [2, pp. 95-97, proof of Satz 6]. 
Let $F$ be a free group of rank 2 and $V$ a free group of greater rank. Denote by $W$ the subgroup of $V$ which is generated by all the elements of the form $(u,(v, u))$ for $u$ and $v$ in $V$. Clearly $W$ is a fully invariant subgroup of $V$, and it is possible to verify that $W<^{2} V$. On the other hand one sees that $W^{(V \rightarrow F)}={ }^{2} V^{(V \rightarrow F)}={ }^{2} F$, and this example indicates how much richer becomes the partially ordered set of all the fully invariant subgroups of a free group as its finite rank increases.

THEOREM 3. The homomorphism $\eta$ of the free group $F$ is an isomorphism if, and only if :

(i) $F^{\eta}$ is a free group.

(ii) No element in the kernel of $\eta$ belongs to a free set of generators of $F$.

Proof. The necessity of these conditions is obvious. Assume conversely that the conditions (i) and (ii) are satisfied by the homomorphism $\eta$. If $\eta$ were not an isomorphism, then the kernel $K$ of $\eta$ would contain an element $x \neq 1$. Clearly $F$ is the free product of two free groups $U$ and $V$ such that $x$ is in $V$ and such that $r(V)$ is finite. We infer from (i) that $F / K$ is a free group, and it follows from Schreier's Theorem that the subgroup $(V K) / K$ of $F / K$ is a free group too. But $(V K) / K$ and $V /(V \cap K)$ are isomorphic groups, showing that the latter group is free. Now we may infer from a result due to $\mathrm{F}$. Levi ${ }^{(38)}$ that $V$ is the free product of free groups $W$ and $Z$ such that $W \cap[V \cap K]=1$ and $Z \leqq V \cap K$. Thus $Z$ is a free factor of $F$. Since $x \neq 1$ is in $V \cap K$, this cross cut is different from 1 . Hence $W \neq V$, and this implies $Z \neq 1$. Thus we have found a free factor $Z \neq 1$ of $F$ which is part of the kernel $K$ of $\eta$. This contradicts (ii), proving that $K=1$ and that $\eta$ is an isomorphism.

CoROLlaRY 3. The homomorphism $\eta$ of the free group $F$ is an isomorphism if, and only if:

(a) $F^{n}$ is a free group.

(b) There exists a characteristic subgroup $C \not F F$ of $F$ such that $\eta$ induces an isomorphism in $F / C$.

Proof. The necessity of the conditions is obvious. If the conditions are satisfied, then the kernel $K$ of $\eta$ is certainly part of $C$. Since $C$ is a characteristic subgroup, different from $F$, it is impossible that $C$ contains elements from a free set of generators of $F$. Thus the conditions (i) and (ii) of Theorem 3 are satisfied by $\eta$, showing that $\eta$ is an isomorphism, as we intended to prove.

III.3. Reduced free groups. If $J$ is a fully invariant subgroup of the free group $F$, then we term the group $R=F / J$ a reduced free group ${ }^{\left({ }^{39}\right)}$.

THEOREM 1. Reduced free groups are self-similar.

(38) This result is contained in the proof of his Eliminationssatz; see footnote 37.

(39) These reduced free groups are equivalent to the $V$-groups as defined by B. Neumann $[1$, p. 512, Definition 9.3]; see the Appendix below. 
This important fact is an immediate consequence of Corollary 1 of II.4 since free groups are self-similar by Theorem 1 of III.1.

COROLlaRY 1. The group $G$ is isomorphic to a retract of the reduced free group $R$ if, and only if, $G$ is similar to $R$ and a homomorphic image of $R$.

This is clearly implied by Theorem 1 and Theorem 2 of II.4.

\section{COROLlARY 2. Retracts of similar reduced free groups are similar.}

This is an obvious inference from Theorem 1 and Corollary 3 of II.4.

The author has not been able to decide the important question whether or not the retracts of reduced free groups are themselves reduced free groups.

The following two facts concerning abelian groups will be needed. Infinite cyclic groups are termed cyclic groups of order 0 ; and the order of an element is always the same as the order of the cyclic group which it generates. If an abelian group is the direct product of cyclic groups of equal order, then the number of direct cyclic factors is the same in any two decompositions and is called the rank of this abelian group.

THEOREM 2. If $R=F / J$, for $J$ a fully invariant subgroup of the free group $F$, is a reduced free group, not 1 , then $R /(R, R)$ is the direct product of $r(F)$ cyclic groups of equal order $o(R),(R, R)=(F, F) J / J=\left[(F, F) F^{\circ}(R)\right] / J$ and $o(R)$ is the 1.c.m. of the orders of the elements in $R$.

We note that $r(F)$ equals therefore the rank $r(R)$ of $R /(R, R)$, and we term $r(R)$ the rank of $R$.

Proof. There exists by Theorem 1 of III.2 one and only one not negative integer $n$ such that $F^{n} \leqq J$ and $(F, F) J=(F, F) F^{n}$. From $J<F$ we deduce $n \neq 1$. From $(R, R)=(F, F) J / J$ we deduce the isomorphy of $R /(R, R)$ and $F /\left[(F, F) F^{n}\right]$, and the latter of these groups is clearly a direct product of $r(F)$ cyclic groups of order $n$, since $F /(F, F)$ is a free abelian group of rank $r(F)$. From $F^{n} \leqq J$ we deduce that the orders of the elements in $R$ are divisors of $n$, and $R$ contains elements of order $n$ since $R /(R, R)$ has been shown to contain elements of order $n$.

Remark $1 . J=(F, F)^{i}$ for $1<i$ is clearly a fully invariant subgroup of the not abelian, free group $F$ so that $R=F / J$ is a reduced free group. Clearly $R /(R, R)$ and $F /(F, F)$ are isomorphic groups so that $o(R)=0$. But the orders of the elements in $(R, R)$ are divisors of $i$, showing that $o(R)=0$ does not imply that every element, not equal to 1 , is of order 0 .

TheOREM 3. A reduced free group $R \neq 1$ may be represented in one and essentially only one way in the form $F / J$ for $J$ a fully invariant subgroup of the free group $F$.

Proof. If $R$ is a reduced free group, then $R=F / J$ for $J$ a suitable fully invariant subgroup of the free group $F$. If $K$ is a fully invariant subgroup of 
the free group $V$ such that $R \sim V / K$, then we infer from Theorem 2 that $r(F)=r(R)=r(V)$, proving the isomorphy of $V$ and $F$. Hence we may assume without loss in generality that $V=F$. Since $F / J$ and $F / K$ are isomorphic groups, we deduce from Corollary 1 of III.1 the existence of an endomorphism $\phi$ of $F$ which induces an isomorphism of $F / J$ upon $F / K$, and hence it follows from (b) of Theorem 6 of II.3 that $K=K^{(F \rightarrow F)} \leqq J$; likewise we see that $J \leqq K$, proving $J=K$. Thus we have shown : To every reduced free group $R \neq 1$ there exists one and essentially only one free group $F$ and exactly one fully invariant subgroup $J$ of $F$ such that $R \sim F / J$; this proves our contention.

TheOREM 4. Suppose that $J \neq F$ is a fully invariant subgroup of the free group $F$, that $N$ is a normal subgroup of the free group $V$, and that $F / J \sim V / N$. Then:

(1) $J^{(F \rightarrow V)} \leqq N$.

(2) If the homomorphism $\phi$ of $F$ into $V$ induces an isomorphism of $F / J$ upon $V / N$, if the homomorphism $\nu$ of $V$ into $F$ induces an isomorphism of $V / N$ upon $F / J$, and if $K$ is the kernel of $\nu$, then:

(2a) $\phi$ is an isomorphism of $F$.

(2b) $F \Phi \cap K=1$.

(2c) $F \sim V / K$.

(3) $J \leqq N^{*}$ for every homomorphism $\kappa$ of $V$ upon $F$.

Proof. (1) is an immediate consequence of Corollary 1 of III.1 and (b) of Theorem 6 of II.3.

If an isomorphism of $F / J$ upon $V / N$ is induced by the homomorphism $\phi$ of $F$ into $V$, then we deduce from Corollary 3 of III.2 that $\phi$ is an isomorphism of $F$ into $V$. If furthermore the homomorphism $\nu$ of $V$ into $F$ induces an isomorphism of $V / N$ upon $F / J$, then the endomorphism $\phi \nu$ of $F$ induces an automorphism of $F / J$, and we deduce from Corollary 3 of III.2 that $\phi \nu$ is an isomorphism of $F$ into $F$. Hence an isomorphism of $F^{\phi}$ into $F$ is induced by $\nu$ in $F^{\phi}$ so that 1 is the cross cut of $F^{\phi}$ and the kernel of $\nu$. Thus we have verified (2a) and (2b). Since $V^{v} \leqq F$, it follows from Schreier's Theorem that $V / K$ is a free group. That $V / K$ and $F$ are isomorphic free groups is now readily deduced from $r(F)=r(F / J)=r(V / K)$, showing the validity of (2c).

If $\kappa$ is a homomorphism of $V$ upon $F$, then $F$ and $V / H$, for $H$ the kernel of $\kappa$, are isomorphic free groups. There exists therefore a subgroup $S$ of $V$ such that $H S=V$ and $H \cap S=1$. This implies in particular that $\kappa$ induces an isomorphism of $S$ upon $F$, and the reciprocal of this isomorphism may be denoted by $\eta$. It follows therefore from (1) that $J^{\eta} \leqq N \cap S$. Since $\eta$ and $\kappa$ effect reciprocal isomorphisms between $F$ and $S$, this implies $J=J^{\eta \kappa} \leqq(N \cap S)^{\star} \leqq N^{\star}$, and this completes the proof.

THEOREM $5\left(^{40}\right)$. If the subgroup $S$ of the free group $F$ contains the fully in-

(40) Neumann [1, p. 512, Theorem 9.1]. 
variant subgroup $J$ of $F$, then the following properties of $S$ imply each other:

(i) $S$ is a fully invariant subgroup of $F$.

(ii) $S / J$ is a fully invariant subgroup of $F / J$.

Proof. If $S$ is a fully invariant subgroup of $F$, and if $\eta$ is an endomorphism of $F / J$, then there exists by Lemma 1 of III.1 and Remark 1 of III.1 an endomorphism $\gamma$ of $F$ which induces $\eta$ in $F / J$. But $S^{\gamma} \leqq S$, since $S$ is fully invariant. Hence $S / J$ is mapped into itself by $\eta$, proving that (ii) is a consequence of (i). If conversely (ii) is satisfied by $S$, and if $\phi$ is an endomorphism of $F$, then $J^{\phi} \leqq J$, since $J$ is fully invariant. Hence an endomorphism of $F / J$ is induced by $\phi$ so that $J S^{\phi} \leqq S$ is a consequence of (ii). Thus $S^{\phi} \leqq S$, completing the proof of the equivalence of (i) and (ii).

Remark 2. The fact that (ii) implies (i) is independent of the hypothesis that $F$ be a free group. But it is easy to construct examples which show that (i) would not imply (ii) if $F$ were not free.

III.4. Similarly reduced free groups. The groups $G$ and $H$ are certainly similar groups if 1 is the only group which is a homomorphic image of both of them. Instances of such pairs of trivially similar groups are rather numerous; we mention only the reduced free groups $F / F^{2}$ and $F / F^{3}$ for $F$ a free group. Thus it seems indicated that we introduce a classification principle more restrictive than that of similarity, and to do this is the object of the present section.

If $H$ and $K$ are any two groups, then we have denoted by $(H \rightarrow K)$ the set of all the homomorphisms of $H$ into $K$. Thus $(H \rightarrow H)$ indicates the set of all the endomorphisms of $H$, and $J^{(H \rightarrow B)} \leqq J$ is the property characteristic for fully invariant subgroups $J$ of $H$.

THEOREM 1. The following properties of the reduced free groups $R$ and $S$, satisfying $0<r(R) \leqq r(S)$, imply each other.

(a) There exist normal subgroups $M$ and $N$ of free groups $H$ and $K$ respectively such that $H / M \sim R, K / N \sim S$ and

$$
M^{(H \rightarrow K)} \leqq N, \quad N^{(K \rightarrow H)} \leqq M .
$$

(b) There exist fully invariant subgroups $J$ and $U$ of free groups $F$ and $V$ respectively such that $F / J \sim R, V / U \sim S$, and

$$
U^{(V \rightarrow F)}=J \text {. }
$$

(c) There exists a fully invariant subgroup $Q$ of a free group $P$ and an idempotent endomorphism $\in$ of $P$ such that $P / Q \sim S$ and $P e / Q^{e} \sim R$.

Proof. We are going to show first that the following condition (d) is a consequence of (a).

(d) There exist fully invariant subgroups $J$ and $U$ of free groups $F$ and $V$ respectively such that $F / J \sim R, V / U \sim S$ and 


$$
J^{(P \rightarrow V)} \leqq U, \quad U^{(V \rightarrow F)} \leqq J .
$$

When proving that (d) is a consequence of (a), we are not going to make any use of the hypothesis $r(R) \leqq r(S)$. Thus we may assume during this proof without loss in generality that $r(H) \leqq r(K)$. It follows now from Theorem 2 of III.2 and Corollary 1 of II.3 that $M=N^{(K \rightarrow H)}$ is a fully invariant subgroup of the free group $H$. Since $S$ is a reduced free group, there exists a representation $S=V / U$ for $U$ a fully invariant subgroup of the free group $V$. From $S \neq 1$ and $r(S)=r(V)$ we deduce immediately that $r(V) \leqq r(K)$. Hence there exists a homomorphism $\kappa$ of $K$ upon $V$, and $U \leqq N^{*}$ is a consequence of (3) of Theorem 4 of III.3. If $\nu$ is some homomorphism of $V$ into $H$, then $\kappa \nu$ is a homomorphism of $K$ into $H$, and we infer $N^{k \nu} \leqq M$ from hypothesis (a). Thus $U^{v} \leqq N^{* v} \leqq M$, proving $U^{(V \rightarrow H)} \leqq M$. Since $K / N$ and $V / U$ are both representations of the same group $S$, we deduce from Corollary 1 of III.1 the existence of a homomorphism $\beta$ of $V$ into $K$ which induces an isomorphism of $V / U$ upon $K / N$. Thus in particular $V^{\beta} \cap N=U^{\beta}$, and it follows from (2a) of Theorem 4 of III.3 that $\beta$ is an isomorphism. If $\eta$ is some homomorphism of $H$ into $V$, then $\eta \beta$ is a homomorphism of $H$ into $K$, and $M^{n \beta} \leqq N$ is a consequence of hypothesis (a). But $M^{\eta \beta}$ is part of $V^{\beta}$, and thus we have shown that $M^{{ }^{\beta}} \leqq V^{\beta} \cap N=U^{\beta}$. Since $\beta$ is an isomorphism, the last inequality implies $M^{n} \leqq U$, proving $M^{(\boldsymbol{B} \rightarrow V)} \leqq U$. Thus (d) is a consequence of (a).

During the remainder of the proof we shall make use of the hypothesis $r(R) \leqq r(S)$.

Assume now that (d) be satisfied by $R$ and $S$. Then it is a consequence of Theorem 2 of III.3 that $r(R)=r(F)$ and $r(S)=r(V)$. Thus it is a consequence of (d), Theorem 2 of III.2 and Corollary 1 of II.3 that $U^{(V \rightarrow F)}=J$. Hence (b) is a consequence of $(d)$ and, therefore, of (a).

Assume now the validity of (b). Then $J^{(F \rightarrow V)}=U^{(V \rightarrow F)(F \rightarrow V)} \leqq U$, since $U$ is a fully invariant subgroup of $V$, showing incidentally the equivalence of (b) and (d) as well as the equivalence of (b) and (a). From Theorem 2 of III.3 we deduce again that $r(F)=r(R) \leqq r(S)=r(V)$, and there exists therefore an idempotent endomorphism $\epsilon$ of $V$ such that $V^{\epsilon}$ and $F$ are isomorphic. (To construct $\epsilon$ consider a free set $B$ of generators of $V$ and a subset $B^{*}$ of $B$ which contains exactly $r(F)$ elements; then $\epsilon$ is the endomorphism of $V$ which leaves invariant all the elements in $B^{*}$ and which maps upon 1 all the elements in $B$, not in $B^{*}$.) Clearly $V^{\bullet} \cap U \leqq U^{\epsilon}$, since $\epsilon=\epsilon^{2}$, and $U^{\epsilon} \leqq V^{\epsilon} \cap U$, since $U$ is fully invariant, proving $U^{e}=V^{\epsilon} \cap U$. There exists an isomorphism $\alpha$ of $F$ upon $V^{e}$. Then $\alpha$ is an isomorphism of $F$ into $V$ too, and we deduce $J^{\alpha} \leqq V^{e} \cap U$ from $J^{(F \rightarrow V)} \leqq U$. Since $\alpha^{-1}$ is an isomorphism of $V^{\epsilon}$ upon $F$, it follows that $\epsilon \alpha^{-1}$ is a homomorphism of $V$ upon $F$. Thus $U^{\epsilon \alpha^{-1}} \leqq J$ is a consequence of $U^{(\nabla \rightarrow F)}=J$. But $\alpha$ and $\alpha^{-1}$ are isomorphisms so that the last inequality implies $V^{\bullet} \cap U=U^{e} \leqq J^{\alpha}$. Consequently we have shown that $U^{\boldsymbol{e}}=J^{\alpha}$; and the isomorphism $\alpha$ of $F$ upon $V^{\epsilon}$ induces therefore an isomorphism of $F / J$ upon $V^{\epsilon} / U^{\epsilon}$, proving that (c) is a consequence of (b). 
Assume finally the validity of (c). Then we verify, as before, that $U^{\epsilon}=V^{\bullet} \cap U$. If $\zeta$ is a homomorphism of $V$ into $V^{\bullet}$, then $\zeta$ is an endomorphism of $V$ and $U^{\zeta} \leqq U \cap V^{\epsilon}=U^{e}$, since $U$ is a fully invariant subgroup of $V$. Hence it follows from (b) of Theorem 2 of II.3 that $U^{\epsilon}=U^{\left(V \rightarrow V^{\epsilon}\right)}$. Thus $U^{\epsilon}$ is a fully invariant subgroup of $V^{e}$, and we have shown that (b) is a consequence of (c). Since we have proved before the equivalence of the properties (a), (d) and (b), this completes the proof.

During the proof of the fact that (c) implies (b) we showed that $J^{\boldsymbol{\epsilon}}=J \cap F^{\epsilon}$, if $J$ is a fully invariant subgroup and $\epsilon$ an idempotent endomorphism of the free group $F$. Since idempotent endomorphisms map the group upon retracts, we deduce from (b) of Theorem 2 of II.3 that $J \cap F^{\epsilon}=J^{\left(F \rightarrow F^{\epsilon}\right)}$. Thus we have established the following fact.

CoROllary 1. If $J$ is a fully invariant subgroup and $\epsilon$ an idempotent endomorphism of the free group $F$, then $J^{\epsilon}=J \cap F^{\epsilon}=J^{\left(F \rightarrow F^{\epsilon}\right)}$ is a fully invariant subgroup of the free group $F^{\epsilon}$.

If the reduced free groups $R \neq 1$ and $S \neq 1$ meet the requirements (a) to (c) of Theorem 1, then they shall be termed similarly reduced free groups.

\section{COROLla RY 2. Similarly reduced free groups are similar.}

Proof. Any two free groups are similar by Theorem 1 of III.1. Thus the similarity of similarly reduced free groups is a consequence of (a) of Theorem 1 and of Coroliary 1 of II.4.

Remark 1. It should be noted that "similar reduced free groups" need not be similarly reduced free groups" (that is, the converse of Corollary 2 is not true), as may be seen from the following example: If $F$ and $V$ are free groups, not 1 , and if $m$ and $n$ are positive integers, not 1 , then $F / F^{m}$ and $V / V^{n}$ are similar reduced free groups, since the normal subgroups $M$ and $N$ of $F$ and $V$ respectively meet the requirements $F^{m} \leqq M, V^{n} \leqq N$ and $F / M \sim V / N$ only if $F^{d} \leqq M$ and $V^{d} \leqq N$ for $d$ the g.c.d. of $m$ and $n$. But $F / F^{m}$ and $V / V^{n}$ are-by (b) of Theorem 1-similarly reduced free groups if, and only if, $m=n$.

Remark 2. The question might be raised why we have not termed the groups $F / M$ and $V / N$ similarly reduced free groups whenever $F$ and $V$ are free groups and $M^{(F \rightarrow V)} \leqq N, N^{(V \rightarrow F)} \leqq M$. This definition would clearly assure similarity of $F / M$ and $V / N$, and it is really a wider concept. But it will become clear in the next section that the admission of groups $F / M$ and $V / N$ which are not reduced free groups would cause inconvenience. That the difference between these two concepts is not very great may be seen from the following considerations: If - as we may assume without loss in generality$r(F) \leqq r(V)$, then we deduce from Theorem 2 of III.2 and Corollary 1 of II.3 that $M=N^{(V \rightarrow F)}$ is a fully invariant subgroup of $F$ so that at least one of the two groups is a reduced free group; and if furthermore $r(F)$ is infinite, then $N=M^{(F \rightarrow V)}$ is a fully invariant subgroup of $V$ so that in this last case both groups are reduced free groups. 
THEOREM 2. If $M$ and $N$ are normal subgroups of the reduced free groups $R \neq 1$ and $S \neq 1$ respectively, if 1 is the only fully invariant subgroup of $R(S)$ which is part of $M(N)$, and if each of the groups $R / M$ and $S / N$ is isomorphic to a subgroup of the other one, then $R$ and $S$ are similarly reduced free groups.

Proof. There exist fully invariant subgroups $J$ and $U$ of free groups $F$ and $V$ respectively such that $R=F / J$ and $S=V / U$. There exist furthermore uniquely determined normal subgroups $M^{*}$ and $N^{*}$ of $F$ and $V$ respectively such that $J \leqq M^{*}, M^{*} / J=M$ and $U \leqq N^{*}, N^{*} / U=N$. We deduce from Theorem 5 of III.3 that $J$ is a greatest fully invariant subgroup of $F$ which is part of $M^{*}$, and that $U$ is a greatest fully invariant subgroup of $V$ which is part of $N^{*}$. There exists an isomorphism of $R / M=F / M^{*}$ into $S / N=V / N^{*}$, and hence we deduce from Lemma 1 of III.1 the existence of a homomorphism of $F$ into $V$ which induces an isomorphism of $F / M^{*}$ into $V / N^{*}$, and likewise we prove the existence of a homomorphism of $V$ into $F$ which induces an isomorphism of $V / N^{*}$ into $F / M^{*}$. Hence it follows from (b) of Corollary 3 of II.3 that $J^{(F \rightarrow V)} \leqq U$ and $U^{(V \rightarrow F)} \leqq J$, and we deduce from (a) of Theorem 1 that $R$ and $S$ are similarly reduced free groups.

The following interesting special cases of Theorem 2 are obtained by putting $M$ and/or $N$ equal to 1 .

COROLlaRy 3. The reduced free groups $R \neq 1$ and $S \neq 1$ are similarly reduced free groups, if they meet either of the following requirements.

(a) Each of the two groups is isomorphic to a subgroup of the other one.

(b) There exists an (idempotent) endomorphism $\epsilon$ of $S$ such that $R \sim S^{\epsilon}$ and such that 1 is the only fully invariant subgroup of $S$ which is part of the kernel of $\epsilon$.

Remark 3. If $G$ and $H$ are free groups of finite or countably infinite rank, not less than 2, then each of them is known to be isomorphic to a subgroup of the other one, though they need not be isomorphic (condition (a)!). It is not known to the author whether or not these are the only instances of reduced free groups which are not isomorphic, though they are each of them isomorphic to a subgroup of the other one.

Remark 4. It is clear that neither of the conditions (a) and (b) is a necessary condition. Condition (b) implies that $R$ is a retract of $S$, but it has been pointed out before that a reduced free group which is a retract of another reduced free group need not be similarly reduced. It would be interesting to find conditions necessary and sufficient for a retract to be similarly reduced.

THEOREM 3. The following properties of the reduced free groups $R \neq 1$ and $S \neq 1$ are equivalent:

(i) $R \sim S$.

(ii) $r(R)=r(S)$ and there exists a reduced free group $T$ of rank not less than $r(R)$ such that both $R, T$ and $S, T$ are pairs of similarly reduced free groups.

(iii) Each of the two groups $R$ and $S$ is a homomorphic image of the other one. 
(iv) $r(R)=r(S)$ and each of the two groups $R$ and $S$ is isomorphic to a subgroup of the other one.

Proof. It is clear that (ii), (iii) and (iv) are consequences of (i). That, conversely, (iv) implies (ii) is immediately deduced from (a) of Corollary 3 . Thus we have to show only that (i) is implied by (ii) as well as by (iii).

If condition (ii) is satisfied by the groups $R$ and $S$, then there exist fully invariant subgroups $J$ and $K$ of a free group $F$ such that $R \sim F / J, S \sim F / K$, and there exists a fully invariant subgroup $U$ of a free group $V$ such that $r(F) \leqq r(V)$ and such that $R, V / U$ and $V / U, S$ are pairs of similarly reduced free groups. But then it follows from (b) of Theorem 1 and from Theorem 3 of III.3 that

$$
J=U^{(\nabla \rightarrow F)}=K,
$$

proving that (i) is a consequence of (ii).

The proof that (i) is a consequence of (iii) shall be preceded by the proof of a lemma.

(3.1) If $J$ and $K$ are fully invariant subgroups of the free groups $F$ and $V$ respectively, and if there exists a homomorphism of $V / K$ upon $F / J$, then $K^{(V \rightarrow F)} \leqq J$.

There exists a normal subgroup $M$ of $V$ which contains $K$ such that $V / M \sim F / J$. Hence it follows from Theorem 1 of III.1 and (b) of Theorem 6 of II.3 that our contention is true.

Assume now that condition (iii) is satisfied by the reduced free groups $R$ and $S$. Then their ranks are clearly equal, and there exist therefore fully invariant subgroups $J$ and $K$ of a free group $F$ such that $R \sim F / J$ and $S \sim F / K$. But now it follows from (3.1) that $J=J^{(F \rightarrow F)} \leqq K=K^{(F \rightarrow F)} \leqq J$, and that consequently $K=J$, proving the desired isomorphy of $R$ and $S$.

Corollary $4\left({ }^{41}\right)$. If $M, R$ and $N, S$ meet the requirements of Theorem 2, and if $r(R)=r(S)$, then $R \sim S$.

This is a fairly immediate consequence of Theorem 2 and of (ii) of Theorem 3.

THEOREM 4. If $R, S, T$ are reduced free groups, not 1 , if $R, S$ as well as $S, T$ are pairs of similarly reduced free groups, and if $r(S)$ is not at the same time finite and smaller than both $r(R)$ and $r(T)$, then $R$ and $T$ are similarly reduced free groups.

Proof. We assume without loss in generality that $r(R) \leqq r(T)$. From our hypotheses we infer the existence of fully invariant subgroups $J$ and $H$ of the free groups $U$ and $V$ respectively such that $R \sim U / J, S \sim V / H$ and

(11) This result is closely connected with certain theorems of B. Neumann [1]; see in particular his Theorem 11.2, p. 514 and his Theorem 12.6, p. 516. 
$J^{(U \rightarrow V)} \leqq H, H^{(V \rightarrow U)} \leqq J$, and the existence of fully invariant subgroups $H^{*}$ and $K$ of the free groups $V^{*}$ and $W$ respectively such that $S \sim V^{*} / H^{*}$, $T \sim W / K$ and $H^{*\left(V^{*} \rightarrow W\right)} \leqq K, K^{\left(W \rightarrow V^{*}\right)} \leqq H^{*}$. It is a consequence of Theorem 3 of III.3 that we may assume without loss in generality $V=V^{*}$ and $H=H^{*}$. We note finally that $r(U)=r(R) \leqq r(T)=r(W)$ is a consequence of Theorem 2 of III.3. We distinguish several cases.

Case 1. $r(R) \leqq r(S) \leqq r(T)$. Then, as before, $r(U) \leqq r(V) \leqq r(W)$. There exists therefore an isomorphism $v$ of $U$ into $V$ and an idempotent endomorphism $\epsilon$ of $V$ such that $U^{v}=V^{\epsilon}$. If $\eta$ is a homomorphism of $W$ into $U$, then $\eta=(\eta v)\left(\epsilon v^{-1}\right)$, since $\epsilon$ affects the identity in $V^{e}$, and since $v^{-1}$ is an isomorphism of $V^{\epsilon}$ upon $U$. But $\eta v$ is a homomorphism of $W$ into $V$ and $\epsilon v^{-1}$ is a homomorphism of $V$ into $U$, proving $(W \rightarrow U)=(W \rightarrow V)(V \rightarrow U)$. From (c) of Theorem 2 of III.2, Corollary 1 of II.3 and $r(U) \leqq r(V) \leqq r(W)$ we infer that $H^{(V \rightarrow U)}=J$ and $K^{(W \rightarrow V)}=H$. Consequently we have $K^{(W \rightarrow U)}=K^{(W \rightarrow V)(V \rightarrow U)}$ $=H^{(V \rightarrow U)}=J$ and $J^{(U \rightarrow W)}=K^{(W \rightarrow U)(U \rightarrow W)} \leqq K$, since $K$ is fully invariant, and this proves that $R$ and $T$ are similarly reduced free groups.

Case 2. $r(R) \leqq r(T) \leqq r(S)$. Then we put $J^{*}=K^{(W \rightarrow U)}$ and $R^{*}=U / J^{*}$. Clearly $R^{*}, T$ and $T, S$ are pairs of similarly reduced free groups, and it follows from Case 1 that $R^{*}$ and $S$ are similarly reduced free groups. But $r(R)=r\left(R^{*}\right)$ and $R$ and $S$ are similarly reduced free groups too. Hence it follows from Theorem 3 that $R$ and $R^{*}$ are isomorphic groups, proving that $R$ and $T$ are similarly reduced free groups.

Case 3. $r(S)<r(R) \leqq r(T)$. It follows from our hypothesis that the three ranks $r(R), r(S)$ and $r(T)$ are infinite. Then it follows from (c) of Theorem 2 of III. 2 and Corollary 1 of II. 3 that $J=H^{(V \rightarrow U)}$ and $H=J^{(U \rightarrow V)}, K^{(W \rightarrow V)}=H$ and $H^{(v \rightarrow W)}=K$. Consequently we deduce from the full invariance of $J, H, K$ that $K=H^{(V \rightarrow W)}=J^{(U \rightarrow V)(V \rightarrow W)} \leqq J^{(U \rightarrow W)}$ and $J=H^{(V \rightarrow U)}=K^{(W \rightarrow V)(V \rightarrow U)} \leqq K^{(W \rightarrow U)}$ $\leqq J^{(U \rightarrow W)(W \rightarrow U)} \leqq J$. Hence $J=K^{(W \rightarrow U)}$, and likewise one sees that $K^{\prime}=J^{(U \rightarrow W)}$, proving again that $R$ and $T$ are similarly reduced free groups.

Remark 5. The indispensability of the condition concerning the ranks of $R, S, T$ may be seen from the following example. Denote by $Z$ an infinite cyclic group and by $F$ a free group of rank 2 . Then both pairs $F, Z$ and $Z$, $F /(F, F)$ are pairs of similarly reduced free groups; but $F$ and $F /(F, F)$ are certainly not similarly reduced free groups, they are not even similar.

We note that the relation of "being similarly reduced free groups," though not transitive, is symmetric and reflexive, as may be deduced from (a) of Theorem 1.

THEOREM 5. If $R, S$ and $T$ are reduced free groups, not 1 , such that $r(S)$ is not smaller than both $r(R)$ and $r(T)$, if $R$ and $S$ are similarly reduced free groups, and if $S$ and $T$ are similar, then $R$ and $T$ are similar.

Proof. If $r(R) \leqq r(S)$, then $R$ is by (c) of Theorem 1 isomorphic to a retract of $S$, and the similarity of $R$ and $T$ is a consequence of Corollary 2 of III.3. 
If $r(S)<r(R)$, then it follows from our hypothesis that $r(T) \leqq r(S)$. Since $R, S$ and $T$ are reduced free groups, there exist fully invariant subgroups $J, K, L$ of free groups $F, V, W$ respectively such that $R \sim F / J, S \sim V / K$ and $T \sim W / L$. Since $r(W) \leqq r(V)<r(F)$ by Theorem 2 of III.3, there exist retracts $F^{*}$ and $V^{*}$ of $F$ and $V$ respectively such that $F^{*} \sim V^{*} \sim W$. Hence there exist normal subgroups $F^{* *}$ and $V^{* *}$ of $F$ and $V$ respectively such that $F=F^{*} F^{* *}, 1=F^{*} \cap F^{* *}$ and $V=V^{*} V^{* *}, 1=V^{*} \cap V^{* *}$. Since $R$ and $S$ are similarly reduced free groups, we deduce from (b) of Theorem 1 (and Theorem 3 of III.3) that $J^{(F \rightarrow V)}=K$. Assume now that $M$ and $N$ are normal subgroups of $F$ and $W$ respectively, that $J \leqq M, L \leqq N$ and $F / M \sim W / N$ (so that $F / M$ and $W / N$ are isomorphic quotient groups of $R$ and $T$ ). There exists an isomorphism $\alpha$ of $W$ upon $F^{*}$. Then $F^{* *} N^{\alpha}$ is a normal subgroup of $F=F^{*} F^{* *}$, since $N^{\alpha}$ is a normal subgroup of $F^{*}$, and $F /\left(F^{* *} N^{\alpha}\right) \sim F^{*} / N^{\alpha} \sim W / N \sim F / M$. Thus it follows from Corollary 1 of III.1 and (b) of Theorem 6 of II.3 that $J \leqq M$ implies $J=J^{(F \rightarrow F)} \leqq F^{* *} N^{\alpha}$. There exists furthermore an isomorphism $\beta$ of $W$ upon $V^{*}$, and as before we see that $V /\left(V^{* *} N^{\beta}\right) \sim W / N$. But $W / N$ and $F /\left(F^{* *} N^{\alpha}\right)$ have been shown before to be isomorphic groups. Thus we deduce from $J \leqq F^{* *} N^{\alpha}$ and Corollary 1 of III.1, (b) of Theorem 6 of II.3, that $K=J^{(F \rightarrow V)} \leqq V^{* *} N^{\beta}$. Hence we have shown that $S \sim V / K$ possesses a quotient group isomorphic to $F / M \sim W / N$. But $R$ and $S$ are similar by Corollary 2 and $S$ and $T$ are similar by hypothesis. Hence the similarity of $R$ and $T$ is a consequence of Corollary 2 of II.4.

Remark 6. The impossibility of completely omitting the hypothesis concerning the ranks of $R, S, T$ may be seen from the example in Remark 5 . Comparing Theorems 4 and 5 one may wonder whether infinity of $r(S)$ would suffice. It is clear that the main step of our proof would break down if $r(S)$ were smaller than both $r(R)$ and $r(T)$. For we had to show that every common homomorphic image of both $R$ and $T$ is a quotient group of $S$, which is clearly impossible to prove if $r(S)$ is smaller than both $r(R)$ and $r(T)$. Still, it is an open question whether or not infinity of $r(S)$ is a sufficient condition.

THEOREM 6. The reduced free groups $R$ and $S$, satisfying $0<r(R) \leqq r(S)$, are similar if, and only if, there exist reduced free groups $T$ and $U$ such that:

(i) $\left.{ }^{42}\right) r(R)=r(T)=r(U)$.

(ii) $R$ and $T$ are both isomorphic to retracts of $U$.

(iii) $S$ and $T$ are similarly reduced free groups.

Proof. Assume first the existence of reduced free groups $T$ and $U$ meeting the requirements (i) to (iii). Then we deduce from (ii) and Corollary 2 of III.3 the similarity of $R$ and $T$; the similarity of $R$ and $S$ is now a consequence of (i), (iii), $r(R) \leqq r(S)$ and Theorem 5 .

Assume conversely the similarity of $R$ and $S$. There exist fully invariant subgroups $J$ and $K$ of free groups $F$ and $V$ respectively such that $F / J \sim R$

(12) It will be seen from the proof that it suffices to assume $0<r(R) \leqq r(T)=r(U) \leqq r(S)$. 
and $V / K \sim S$. Put $L=K^{(V \rightarrow F)}$. Then we deduce from $r(R) \leqq r(S)$ and from (b) of Theorem 1 that $F / L=T$ and $S$ are similarly reduced free groups, and it is a consequence of (c) of Theorem 1 that $T$ is a retract of $S$. Thus the reduced free groups $T$ and $R$ are retracts of the similar reduced free groups $S$ and $R$ respectively, and it follows from Corollary 2 that $T$ and $R$ are similar. Consequently we may deduce from Theorem 1 of III.3 and (iii) of Theorem 4 of II.4 that $T=F / L$ and $[R \sim] F / J$ are isomorphic to retracts of the reduced free group $U=F /(L \cap J)$. It is clear now that the reduced free groups $T$ and $U$ meet the requirements (i) to (iii), completing the proof.

The following theorem is a partial extension of Theorem 2 of III.1 from free groups to reduced free groups.

THEOREM 7. If $M$ and $N$ are normal subgroups of the reduced free group $R$ such that $M \leqq N$ and such that $R / N$ is a not redundant representation, then there exist a reduced free group $Q$ and a normal subgroup $L$ of $Q$ with the following properties.

(a) $R$ and $Q$ are similarly reduced free groups.

(b) $R / M \sim Q / L$.

(c) There exists a homomorphism $\gamma$ of $Q$ satisfying $Q^{\gamma}=R$ and $L^{\gamma}=N$.

Proof. There exists one (and essentially only one) representation $R=F / J$ for $J$ a fully invariant subgroup of the free group $F$, and there exist uniquely determined normal subgroups $M^{*}$ and $N^{*}$ of $F$ which satisfy $J \leqq M^{*} \leqq N^{*}$ and $M^{*} / J=M, N^{*} / J=N$. Since the representation $R / N$ is not redundant, it follows that the representation $F / N^{*}$ of $R / N$ and the representation $F / M^{*}$ of $R / M$ are not redundant.

Denote by $V / L^{*}$ the regular representation of the group $R / M$. Since $R / N$ is a homomorphic map of the group $R / M$, there exists by Theorem 2 of III.1 a homomorphism $\nu$ of $V$ such that $V^{\nu}=R$ and $L^{*_{\nu}}=N$. We deduce from Lemma 1 of III.1 the existence of a homomorphism $\phi$ of $V$ into $F$ which induces the homomorphism $\nu$ of $V$ upon $R=F / J$. From $V / L^{*} \sim R / M$ $\sim F / M^{*}$ and Corollary 1 of III.1 we deduce the similarity of the representations $V / L^{*}$ and $F / M^{*}$ of the group $R / M$. Hence it follows from (b) of Theorem 6 of II.3 that $K=J^{(F \rightarrow V)} \leqq L^{*}$. Clearly $K$ is a fully invariant subgroup of the free group $V$, satisfying $K^{(V \rightarrow F)} \leqq J$. Consequently $R$ and $Q=V / K$ are similarly reduced free groups. Furthermore $L=L^{*} / K$ is a normal subgroup of $Q$ such that $Q / L \sim V / L^{*} \sim R / M$. Finally we note that $K^{\phi} \leqq J$ and that $\phi$ induces therefore a homomorphism $\gamma$ of $Q$ into $R$. But $\gamma$ is clearly the homomorphism induced by $\nu$ in $Q=V / K$ so that $Q^{\gamma}=R$ and $L^{\gamma}=N$, completing the proof.

We note that the representation $Q / L$ of $R / M$ constructed in the course of the proof might be termed a "reduced regular representation."

III.5. Complete systems of similarly reduced free groups. We precede our discussion by the proof of two useful lemmas. 
LEMMA 1. If $R \neq 1$ is a reduced free group, if $c$ is a cardinal number $\left({ }^{43}\right)$, not 0 , and if $r(R)$ is not at the same time finite and smaller than $c$, then there exists one and essentially only one reduced free group $S$ such that $r(S)=c$ and such that $R$ and $S$ are similarly reduced free groups.

Proof. $R=F / J$ for $J$ a fully invariant subgroup of the free group $F$. Denote by $V$ a free group of rank $c$ and put $K=J^{(F \rightarrow V)}$. Then $K^{(V \rightarrow F)}=J^{(P \rightarrow V)(V \rightarrow P)} \leqq J$. Hence $S=V / K$ is a reduced free group of rank $c$, and $R$ and $S$ are clearly similarly reduced free groups ((a) of Theorem 1 of III.4).

Suppose now that $T$ and $R$ are similarly reduced free groups and that $r(T)=c$. Then it follows from our hypothesis concerning $c$ and $r(R)$, and from Theorem 4 of III.4, that $S$ and $T$ are similarly reduced free groups of equal rank, and hence $S$ and $T$ are isomorphic groups by Theorem 3 of III.4.

Remark 1. We note that the hypothesis concerning $c$ and $r(R)$ has been used only in the proof of the unicity statement. That this hypothesis is indispensable for the proof of the unicity statement has been pointed out before (Remark 2 of III.2).

LEMMA 2. If $P$ is a system of similarly reduced free groups whose ranks are finite, but not bounded, then there exists one and essentially only one reduced free group $R$ of rank $\aleph_{0}$ such that $R$ and $S$, for every $S$ in $P$, are similarly reduced free groups.

Proof. It is a consequence of Lemma 1 (or of Theorem 3 of III.4) that $P$ contains at most one reduced free group of any given finite rank $i$ (apart from isomorphic ones which we do not consider different). Hence it is possible to number the groups in $P$ in such a way that $P$ contains a group $R_{i}$ for every positive integer $i$ and that $r\left(R_{i}\right)<r\left(R_{i+1}\right)$ for $0<i$. Clearly $R_{i}=F(i) / J(i)$ for $J(i)$ a fully invariant subgroup of the free group $F(i)$ and $r(F(i))=r\left(R_{i}\right)$. If we put $K(i)=J(i)^{(F(i) \rightarrow F)}$ for $F$ a free group of $\operatorname{rank} \aleph_{0}$, then $K(i)^{(F \rightarrow F(i))} \leqq J(i)$ and it follows from (a) of Theorem 1 of III.4 that $R_{i}$ and $F / K(i)$ are similarly reduced free groups. We deduce from Theorem 4 of III.4 that $R_{i-1}$ and $F / K(i)$ are similarly reduced free groups, and from this fact we infer-because of Theorem 3 of III.3-that $K(i-1) \leqq K(i)$. We denote by $J$ the (settheoretical) join of the subgroups $K(i)$ of $F$. It is clear that $J$ is a fully invariant subgroup of $F$; and that $J(i)^{(F(i) \rightarrow F)} \leqq J$. If $x$ is an element in $J$, and if $i$ is a given integer, then there exists an integer $j$, not less than $i$, such that $x$ belongs to $K(j)$. Since $F / K(j), R_{j}$ and $R_{j}, R_{i}$ are pairs of similarly reduced free groups, and since $r\left(R_{i}\right)<r\left(R_{j}\right)<r(F / K(j))$, it follows from Theorem 4 of III.4 that $R_{i}$ and $F / K(j)$ are similarly reduced free groups, implying $K(j)^{(F \rightarrow F(i))} \leqq J(i)$. Thus in particular $x^{(F \rightarrow F(i))} \leqq J(i)$ for every $x$ in $J$, or $J^{(F \rightarrow F(i))} \leqq J(i)$, proving that $R_{i}$ and $F / J=R$ are similarly reduced free groups.

If $S$ is any reduced free group of rank $\aleph_{0}$ such that $S$ and $R_{i}$ are similarly

(43) Cardinal numbers may be finite or infinite. 
reduced free groups for every $i$, then $S$ may be represented in the form $F / K$ for $K$ a suitable fully invariant subgroup of $F$. We deduce $K^{(F \rightarrow F(i))}=J(i)$ from (b) of Theorem 1 of III.4 and Theorem 3 of III.3, proving $K(i) \leqq K$ for every $i$. Hence $J \leqq K$. If $x$ is an element in $K$, then there exists a retract $F^{*}$ of $F$ which contains $x$ and which satisfies $r\left(F^{*}\right)=r(F(i))$, for suitable $i$, since the finite ranks $r(F(i))$ are not bounded. There exists a homomorphism $\eta$ of $F$ upon $F(i)$ which induces an isomorphism $\alpha$ of $F^{*}$ upon $F(i)$. Hence $x^{\alpha}=x^{\eta}$ belongs to $J(i)$. But $\alpha^{-1}$ is an isomorphism of $F(i)$ into $F$. Hence $x=x^{\alpha^{-1}}$ belongs to $J(i)^{(F(i) \rightarrow F)}=K(i) \leqq J$, and thus we have shown $K \leqq J$ too. Hence $J=K$, proving the isomorphy of $R$ and $S$. This completes the proof.

If $P$ is a system of similarly reduced free groups, that is, if any two groups in $P$ are similarly reduced free groups, then $P$ contains at most one reduced free group of any given rank, if isomorphic groups in $P$ are not considered different (Lemma 1). Thus a maximal system of similarly reduced free groups would be one that contains reduced free groups of all ranks. Since such a definition might lead us into a controversial set-theoretical situation, we prefer the following slightly more general concept.

A c-complete system, for $c$ a (finite or infinite) cardinal number, is a set $\Xi$ of groups meeting the following requirements.

(1) Every group in $\Xi$ is a reduced free group of rank smaller than $c$.

(2) contains groups of every rank smaller than $c$.

(3) Any two groups in $\mathbf{\Xi}$ are similarly reduced free groups.

Because of (3) and Lemma 1 it is impossible that $\Xi$ contains essentially different groups of any given rank. If we identify isomorphic groups in $\boldsymbol{\Xi}$, then (2) may be restated in the following equivalent form.

$\left(2^{\prime}\right)$ I contains one and only one group of every rank smaller than $c$.

The existence of $c$-complete systems will be assured by the following:

Theorem 1. Suppose that $P$ is a system of similarly reduced free groups, not 1 , and that the cardinal number $c$ is greater than the rank of every group in $P$.

(a) $P$ is contained in some c-complete system.

(b) $P$ is contained in one and essentially only one c-complete system, if either:

$\left(\mathrm{b}^{\prime}\right) c$ is finite and $P$ contains a group of rank $c-1$.

$\left(b^{\prime \prime}\right) c$ is infinite and the ranks of the groups in $P$ are not bounded by a finite number.

Proof. Consider the class of all the systems $Q$ meeting the following requirements :

(i) $P$ is part of $Q$.

(ii) The elements in $Q$ are reduced free groups of rank smaller than $c$.

(iii) Any two groups in $Q$ are similarly reduced free groups.

It is readily seen that this class contains a maximal system $Q_{0}$ (maximumprinciple) $\left({ }^{44}\right)$. Denote by $m$ any cardinal number less than $c$.

(4) Teichmüller [1], Zorn [1]. 
Case $1 . Q_{0}$ contains a group $R$ such that $r(R)$ is not at the same time finite and smaller than $m$.

Then we deduce from Lemma 1 the existence of one and essentially only one reduced free group $S$ of rank $m$ such that $R$ and $S$ are similarly reduced free groups. If $X$ is any reduced free group in $Q_{0}$, then both $X, R$ and $R, S$ are pairs of similarly reduced free groups, and we deduce from Theorem 4 of III.4 that $X$ and $S$ are similarly reduced free groups. The system consisting of the groups in $Q_{0}$ and of the group $S$ meets therefore the requirements (i) to (iii), proving that $S$ is isomorphic to a group in $Q_{0}$.

Case 2. The rank of every group $R$ in $Q_{0}$ is finite and smaller than $m$. It will be convenient to distinguish two subcases.

Case 2.1. The ranks of the groups in $Q_{0}$ are bounded by a finite number. Then there exists a group $G$ of greatest rank in $Q_{0}$. Clearly $r(G)<m$, and we deduce from Lemma 1 (see Remark 1) the existence of a group $T$ of rank $m$ such that $G$ and $T$ are similarly reduced free groups. If $X$ is any group in $Q_{0}$, then $r(X) \leqq r(G)<r(T)$ and both $X, G$ and $G, T$ are pairs of similarly reduced free groups. Hence it follows again from Theorem 4 of III.4 that $X$ and $T$ are similarly reduced free groups, and we show, as in Case 1 , that $Q_{0}$ contains a group isomorphic to $T$, a contradiction.

Case 2.2. The ranks of the groups in $Q_{0}$ are not bounded by a finite number. Then $m$ is necessarily infinite. We deduce from Lemma 2 the existence of one and essentially only one group $H$ of $\operatorname{rank} \boldsymbol{\aleph}_{0}$ such that $Z$ and $H$ are similarly reduced free groups for every $Z$ in $Q_{0}$. Thus it follows, as in Case 1 , that $Q_{0}$ contains a group isomorphic to $H$, a contradiction.

Thus we have shown that $Q_{0}$ contains reduced free groups of every rank $m<c$, proving that $Q_{0}$ is a $c$-complete system, containing $P$.

Suppose now that $Q_{0}$ and $\Xi$ are $c$-complete systems, containing $P$. If $P$ contains a group $R$ which is either of infinite rank or, in case $c$ is finite, of rank $c-1$, then we infer from Lemma 1 that the groups of rank $m<c$ in $Q_{0}$ and $\Xi$ respectively are isomorphic, proving that $Q_{0}$ and $\Xi$ are essentially the same. If the groups in $P$ are all of finite rank, but their ranks are not bounded by a finite number, then we deduce from Lemma 2 that $Q_{0}$ and $\Xi$ contain isomorphic groups of rank $\boldsymbol{\aleph}_{0}$, provided $\boldsymbol{\aleph}_{0}<c$, and their essential identity is proven as before. If $c=\boldsymbol{N}_{0}$, and if the ranks of the groups in $P$ are not bounded, then we deduce from Lemma 1 that $Q_{0}$ and $\Xi$ do not differ substantially. Thus $Q_{0}$ and $\Xi$ are essentially the same if conditions $\left(\mathrm{b}^{\prime}\right)$ or $\left(\mathrm{b}^{\prime \prime}\right)$ are satisfied by $P$, and this completes the proof of our theorem.

That the conditions $\left(b^{\prime}\right)$ and $\left(b^{\prime \prime}\right)$ are not necessary may be seen from the following fact.

THEOREM 2. If the c-complete system $\Xi$ contains a free group of rank not less than 2 , then every group in $\Xi$ is free.

Proof. Suppose that $F$ is a free group of rank not less than 2, that $J$ is a 
fully invariant subgroup of the free group $V$, and that $F$ and $V / J$ are similarly reduced free groups. Then $J^{(V \rightarrow F)}=1$, and we deduce $J=1$ from Theorem 2 of III. 2 and Theorem 3 of II.3. Our theorem is an immediate consequence of this fact.

THEOREM 3. The group $G$ is a homomorphic image of a group in the c-complete system $\Xi$ if, and only if:

(i) $G$ may be generated by less than $c$ elements.

(ii) There exists a reduced free group $R$ such that $G$ is a homomorphic image of $R$ and such that $R$ and $S$, for every $S$ in $\Xi$, are similarly reduced free groups.

Proof. The necessity of the conditions being obvious, let us assume that conditions (i) and (ii) are satisfied by $G$. Then there exists a free group $F$ of rank less than $c$, a normal subgroup $N$ of $F$ and a fully invariant subgroup $J$ of $F$ such that $F / N \sim G$ and such that $F / J$ belongs to $\Xi$. There exists furthermore a free group $V$, a normal subgroup $M$ of $V$ and a fully invariant subgroup $L$ of $V$ such that $V / M \sim G, L \leqq M$ and such that $F / J$ and $V / L$ are similarly reduced free groups. If $r(V) \leqq r(F)$, then $V / L$ belongs to $\Xi$ by Lemma 1 , and $G$ has been shown to be a homomorphic image of a group in $\Xi$. If $r(F)<r(V)$, then we infer from (b) of Theorem 1 of III.4 and Theorem 3 of III.3 that $L^{(V \rightarrow F)}=J$ and $J^{(F \rightarrow V)} \leqq L$, and we deduce from (b) of Theorem 6 of II.3 that $J=L^{(V \rightarrow F)} \leqq N$, since $V / M \sim F / N$. Thus $G$ is a homomorphic image of the group $F / J$ in $\Xi$, completing the proof.

If $\Xi$ is a $c$-complete system, then $\Xi_{h}$ is the system of all the homomorphic images of groups in $\Xi$, and it has been pointed out in Corollary 4 of II.4 that the representations of a group $G$ in $\Xi_{h}$ in the form $F / N$ for $F$ in $\Xi$ form one and only one class of similar representations of $G$. Since every class of groups is equivalent to a group theoretical property, it seems important to characterize the systems $\boldsymbol{\Xi}_{h}$ by inner properties, and not only as a derivate from the $c$-complete system $\boldsymbol{\Xi}$.

We precede the existence theorem by the following uniqueness theorem.

TheOREM 4. If $\Xi$ and $\Sigma$ are c-complete systems such that $\Xi_{h}=\Sigma_{h}$, then $\Xi=\Sigma$.

Proof. If $m$ is a cardinal number smaller than $c$, then $\Xi$ contains by Lemma 1 one and only one reduced free group $R$ of rank $m$, and $\Sigma$ contains likewise one and only one reduced free group $S$ of rank $m$. But $R$ belongs to $\Sigma_{h}$ and is therefore a homomorphic image of some group in $\Sigma$. Thus it follows from Theorem 3 and its proof that $R$ is a homomorphic image of $S$, and likewise one shows that $S$ is a homomorphic image of $R$. Hence it follows from Theorem 3 of III.4 that $R$ and $S$ are isomorphic, proving our contention.

THEOREM 5. The system $\Sigma$ of groups is of the form $\Sigma=\Xi_{h}$ for $\Xi$ a suitable c-complete system if, and only if, $\Sigma$ meets the following requirements. 
(i) Every group in $\Sigma$ may be generated by less than c elements.

(ii) $\Sigma=\Sigma_{h}$.

(iii) If the normal subgroup $N$ of the group $G$ does not contain any fully invariant subgroup of $G$ excepting 1 , if $G$ may be generated by less than $c$ elements, and if $G / N$ belongs to $\Sigma$, then $G$ belongs to $\Sigma$.

(iv) If $\Pi$ is a subset of $\Sigma$, and if there exists a cardinal number $m<c$ such that every group in $\Pi$ may be generated by not more than $m$ elements, then there exists a group $P$ in $\Sigma$ such that every group in $\Pi$ is a homomorphic image of $P$.

Proof. If $\Sigma=\Xi_{h}$ for $\Xi$ a $c$-complete system, then the validity of (i) and (ii) is obvious. If the group $G$ may be generated by less than $c$ elements, then $G=F / M$ where $F$ is a free group of rank less than $c$. Since $\Xi$ is $c$-complete, there exists a uniquely determined fully invariant subgroup $J$ of $F$ such that $F / J$ belongs to $\Xi$. If $N$ is a normal subgroup of $G$, then denote by $N^{*}$ the uniquely determined normal subgroup of $F$ which contains $M$ and satisfies $N=N^{*} / M$. If $N$ does not contain any fully invariant subgroup of $G$ excepting 1 , then we infer from Lemma 1 of III.1 that every fully invariant subgroup of $F$ which is part of $N^{*}$ is contained in $M$. If $G / N$ belongs to $\Sigma$, then there exists a free group $V$, a normal subgroup $H$ of $V$ and a fully invariant subgroup $K$ of $V$ such that $V / K$ is in $\Xi, K \leqq H$, and $V / H \sim G / N \sim F / N^{*}$. Since both $V / K$ and $F / J$ belong to $\Xi$, they are similarly reduced free groups and $K^{(V \rightarrow F)} \leqq J, J^{(F \rightarrow V)} \leqq K$ may be inferred from Theorem 1 of III.4 and Theorem 3 of III.3. We infer now from the isomorphy of $V / H$ and $F / N^{*}$, from Corollary 1 of III.1 and (a) of Theorem 6 of II.3 that $J \leqq N^{*}$. But $J$ is a fully invariant subgroup of $F$, proving $J \leqq M$. Thus $G=F / M$ is a quotient group of the reduced free group $F / J$ in $\Xi$, showing that $G$ belongs to $\Sigma$. Hence we have verified the necessity of condition (iii). Suppose now that $\Pi$ is a subset of $\Sigma$, that $m<c$ is a cardinal number and that every group in $\Pi$ may be generated by not more than $m$ elements. There exists in $\Xi$ one and only one reduced free group $R$ of rank $m$. If $G$ is a group in $\Pi$, then it may be generated by not more than $m$ elements, and $G$ is a homomorphic image of a group $S$ in $\Xi$. Then we deduce from Theorem 3 the existence of a reduced free group $S^{*}$ in $\Xi$ such that $r\left(S^{*}\right) \leqq m$ and such that $G$ is a homomorphic image of $S^{*}$. It is a consequence of (c) of Theorem 1 of III.4 and of the fact that $R$ and $S^{*}$ are similarly reduced free groups that $S^{*}$ is a homomorphic image of $R$. Hence $G$ is a homomorphic image of $R$ too, proving the necessity of condition (iv).

We assume conversely that the conditions (i) to (iv) are satisfied by the system $\Pi$. If $G$ is any group in $\Pi$, then $G$ admits because of (i) a representation $G=F / N$ where $N$ is a normal subgroup of the free group $F$ and where $r(F)<c$. Denote by $J$ the (uniquely determined) greatest fully invariant subgroup of $F$ which is part of $N$. The reduced free group $F / J$ is of rank less than $c$. It follows furthermore from Theorem 5 of III.3 that every fully 
invariant subgroup of $F / J$ which is part of $N / J$ is equal to 1 . Thus it follows from condition (iii) that the reduced free group $F / J$ (of which $G=F / N$ is a homomorphic image) belongs to $\Pi$. We restate the result thus obtained.

(a) Every group in $\Pi$ is a homomorphic image of a reduced free group in $\Pi$.

Denote by $\Pi_{m}$, for $m \neq 0$ a cardinal number smaller than $c$, the set of all the reduced free groups of rank $m$ which are contained in II. If $F_{m}$ is a free group of rank $m$, then it follows from Theorem 3 of III.3 that every group in $\Pi_{m}$ may be represented in one and only one way in the form $F_{m} / J$ for $J$ a fully invariant subgroup of $F_{m}$. It is a consequence of condition (iv) and of (a) that there exists a reduced free group $R$ in $\Pi$ of which every group in $\Pi_{m}$ is a homomorphic image. Obviously $m \leqq r(R)$. There exists by Lemma 1 one and only one reduced free group $S$ of rank $m$ such that $R$ and $S$ are similarly reduced free groups, and from $r(S) \leqq r(R)$ and (c) of Theorem 1 of III.4 it follows that $S$ is a homomorphic image of $R$. Thus we deduce from condition (ii) that $S$ belongs to $\Pi_{m}$. Let $R=V / U$ and $S=F_{m} / J_{m}$ where $J_{m}$ is a uniquely determined fully invariant subgroup of $F_{m}$ and where $U$ is a fully invariant subgroup of the free group $V$. Then $U^{\left(V \rightarrow F_{m}\right)}=J_{m}$. If $G$ is any group in $\Pi_{m}$, then there exists a fully invariant subgroup $J$ of $F_{m}$ and a normal subgroup $N$ of $V$ such that $U \leqq N$ and $V / N \sim F_{m} / J \sim G$. Hence it follows from Corollary 1 of III.1 and (b) of Theorem 6 of II.3 that $J_{m} \leqq J$. Thus the following fact may be deduced from condition (ii).

(b) If $m \neq 0$ is a cardinal number smaller than $C$, then there exists a fully invariant subgroup $J_{m}$ of the free group $F_{m}$ such that $\Pi_{m}$ is exactly the set of all the reduced free groups $F_{m} / J$ for $J$ any fully invariant subgroup of $F_{m}$ satisfying $J_{m} \leqq J$.

Suppose now that $m$ and $n$ are two cardinal numbers satisfying $0<m<n$ $<c$. Put $\mathrm{A}=\left(F_{m} \rightarrow F_{n}\right)$ and $\mathrm{B}=\left(F_{n} \rightarrow F_{m}\right)$. Then it follows from (c) of Theorem 1 of III.4 that $F_{m} / J_{n}^{\mathrm{B}}$ is a homomorphic image of $F_{n} / J_{n}$, and belongs therefore as a consequence of condition (ii) to $\Pi_{m}$. Applying (b) we deduce $J_{m} \leqq J_{n}^{\mathbf{B}}$ so that $J_{m}^{\mathrm{A}} \leqq J_{n}^{\mathrm{BA}} \leqq J_{n}$. There exists a homomorphism $\eta$ of $F_{n}$ upon the whole group $F_{m}$. If $N$ is the normal subgroup of $F_{n}$ consisting of all the elements which are mapped by $\eta$ upon elements in $J_{m}$, then $F_{m} / J_{m} \sim F_{n} / N$ belongs to $\Pi$. If $W$ is the uniquely determined greatest fully invariant subgroup of $F_{n}$ which is part of $N$, then we deduce from condition (iii) that $F_{n} / W$ belongs to $\Pi_{n}$. Hence it follows from (b) that $J_{n} \leqq W \leqq N$. Consequently we deduce from Corollary 1 of III.1 and (b) of Theorem 6 of II.3 that $J_{n}^{\mathrm{B}} \leqq J_{m}$. Thus $F_{m} / J_{m}$ and $F_{n} / J_{n}$ are similarly reduced free groups; and we have shown:

(c) The set $\Xi$ of the reduced free groups $F_{m} / J_{m}$, for $m$ a cardinal number smaller than $c$ and $F_{m}, J_{m}$ defined as in (b), is a $c$-complete system.

But it is an immediate consequence of (a) and (b) that $\Pi=\Xi_{h}$, and this completes the proof of our theorem.

Remark 2. The set of all the homomorphic images of a given group $G$ 
meets, for suitably chosen $c$, the requirements (i), (ii) and (iv), but not the requirement (iii), unless $G$ is a reduced free group, showing the impossibility of omitting condition (iii).

Remark 3. The class $P_{c}$ of all the groups generated by less than $c$ elements the orders of whose elements are divisors of a suitable positive number (which may change from group to group) meets the requirements (i) to (iii), though it cannot satisfy (iv), since 1 is the cross cut of the groups $F^{n}$, for $F$ a free group and $n$ ranging over all the positive integers, showing the impossibility of omitting condition (iv).

Appendix on word subgroups and identical relations. The following way of defining "word subgroups" ${ }^{45}$ ) seems to be most convenient for connecting these concepts with the concepts discussed here. Denote by $W$ a free group of countably infinite rank. If $G$ is any group, and if $S$ is a subset of $W$, then $S^{(W \rightarrow G)}$ is the word subgroup of $G$ defined by $S$. Clearly every word subgroup of the group $G$ is a fully invariant subgroup of $G$. If $G$ is a free group (or a reduced free group), then one infers readily from the results of \$III.2 the well known theorem( $\left.{ }^{46}\right)$ that all the fully invariant subgroups of $G$ are word subgroups. Likewise one sees easily that the reduced free groups $F / J$ and $V / K$, for $J$ and $K$ fully invariant subgroups of the free groups $F$ and $V$ respectively, are similarly reduced free groups if, and only if, $J$ and $K$ may as word subgroups be defined by the same subset of $W$, and that is equivalent to saying that the same "identical relations" ${ }^{47}$ ) are satisfied in $F / J$ and $V / K$. One deduces finally that the system $\Sigma$ of groups is exactly the class of all the groups which may firstly be generated by less than $c$ elements and which secondly all satisfy the identical relations from a certain subset $S$ in $W$ if, and only if, $\Sigma=\Xi_{h}$ for $\Xi$ a suitable $c$-complete system.

III.6. Representations with interlocking lower central series. If any of the $p$ - $N$-series of the group $G$ is interlocking (for concepts see $\S I .2$ ), then $N \leqq(G, G)$. If conversely $N \leqq(G, G)$, then it follows from Theorem 3 of $\mathbf{I . 3}$ that at least the lower central series relative to $N$ is interlocking. We have shown furthermore that interlocking $p$ - $N$-series lead to more invariants than ordinary series (Theorem 2 of II.2). Thus we are led to the problem of representing a given group in the form $G / N$ with $N \leqq(G, G)$. Since the trivial representation of every group has this property, we have to restrict the range of groups $G$ admitted, and thus we ask for representations of a given group in the form $R / M$ for $M$ a normal subgroup of the reduced free group $R$, satisfying $M \leqq(R, R)$.

THEOREM. The group $G \neq 1$ may be represented in the form $R / M$ where $R$ is a reduced free group and where the normal subgroup $M$ of $R$ is part of the commutator subgroup $(R, R)$ of $R$ if, and only if:

(45) Levi [2, pp. 92, 93]; Neumann [1, p. 510, Definition 6.1].

(46) Neumann $[1$, p. 512, Theoren 9.1].

(47) Neumann [1]. 
(i) $G /(G, G)$ is the direct product of cyclic groups of equal order $o(G) \neq 1$.

(ii) $o(G)$ is the l.c.m. of the orders of the elements in $G$.

(iii) There exists a set of generators of $G$ which represents exactly a basis of $G /(G, G)$.

Proof. Assume first that $G=R / M$ where $M$ is a normal subgroup of the reduced free group $R$ and where $M \leqq(R, R)$. It is a consequence of Theorem 2 of III.3 that $R /(R, R)$ is the direct product of $r(R)$ cyclic groups of equal order $o(R)$ and that $o(R)$ is the l.c.m. of the orders of the elements in $R$. But $R /(R, R)$ and $G /(G, G)$ are isomorphic groups, since $M \leqq(R, R)$, and the "natural" isomorphism between $R /(R, R)$ and $G /(G, G)$ proves immediately that (i) and (ii) are satisfied by $G$. The necessity of (iii) is verified likewise, since it is easy to see that property (iii) is satisfied by all reduced free groups.

Suppose conversely that the conditions (i) to (iii) are satisfied by $G$. Then there exists a set $D$ of generators of $G$ which represents exactly a basis of $G /(G, G)$. This implies the existence of a free group $F$, a free set $B$ of generators of $F$ and a homomorphism $\phi$ of $F$ upon $G$ which effects a 1:1 correspondence between $B$ and $D$. The kernel of $\phi$ may be denoted by $M$.

Since $o(G)$ is the l.c.m. of the orders of the elements in $G$, we have $G^{o(}(\theta)=1$, and this implies

$$
F^{\circ}(G) \leqq M .
$$

Since $B$ represents exactly a basis of $F /(F, F)$, it is possible to write every element $x$ in $F$ in the form:

$$
x=x^{\prime} b(1)^{n(1)} \cdots b(k)^{n(k)}
$$

where $x^{\prime}$ is in $(F, F)$ and where the $b(i)$ are distinct elements in $B$. Clearly $x^{\phi}=x^{\prime \phi} d(1)^{n(1)} \cdots d(k)^{n(k)}$ where $x^{\prime \phi}$ belongs to $(G, G)$, and where the elements $d(i)=b(i)^{\phi}$ are distinct elements in $D$. If $x$ happens to belong to $M$, then $x^{\phi}=1$; and we deduce from the fact that $D$ represents exactly a basis of $G /(G, G)$ the fact that $o(G)$ is a divisor of all the $n(i)$. Thus we have shown that $x$ is in $(F, F) F^{\circ(G)}$, whenever $x$ is in $M$; or

$$
M \leqq(F, F) F^{\circ}(G) \text {. }
$$

We put now $R=F / F^{o(\theta)}$ so that $R$ is a reduced free group, namely the free group reduced modulo $o(G)$, and we put $N=M / F^{o(G)}$, which is possible because of (1). Clearly $R / N \sim F / M=G$ and $N=M / F^{\circ(G)} \leqq\left[(F, F) F^{\circ(G)}\right] / F^{\circ}(G)$ $=(R, R)$ because of $(2)$. Thus we have shown the sufficiency of the conditions (i) to (iii). As a matter of fact we have shown slightly more, namely the following theorem.

CoROLlaRY. If conditions (i) to (iii) of the theorem are satisfied by the group $G$, then $G=R / N$ where $R$ is the free group reduced modulo $o(G)$ and where $N \leqq(R, R)$. 
Remark 1. If $o(G)$ in condition (i) happens to be 0 , then $G /(G, G)$ is a free abelian group and condition (ii) may be omitted.

Remark 2. Condition (iii) does not imply that every set of elements in $G$ which represents exactly a basis of $G /(G, G)$ generates the group $G$; as an example to the contrary take for $G$ any not abelian free group. It should be remembered, however, that the property just mentioned may serve in the case of finite groups as a characteristic property of nilpotent groups $\left({ }^{48}\right)$. Consequently the finite nilpotent groups with the property that the orders of all their elements are squarefree meet all the requirements of the theorem.

\section{R. BAER}

\section{BiBLIOGRAPHY}

1. Erweiterung von Gruppen und ihren Isomorphismen, Math. Zeit. vol. 38 (1934) pp.375416.

2. Nilpotent groups and their generalizations, Trans. Amer. Math. Soc. vol. 47 (1940) pp. 393-434.

3. The higher commutator subgroups of a group, Bull. Amer. Math. Soc. vol. 50 (1944) pp. 143-160.

4. Representations of groups as quotient groups. II. Minimal central series. Trans. Amer. Math. Soc. vol. 58 (1945) pp. 348-389.

5. Representations of groups as quotient groups. III. Trans. Amer. Math. Soc. vol. 58 (1945) pp. 390-419.

S. Eilenberg and S. MacLane

1. Group extensions and homology, Ann. of Math. vol. 43 (1942) pp. 757-831.

2. Natural isomorphisms in group theory, Proc. Nat. Acad. Sci. U.S.A. vol. 28 (1942) pp. 537-543.

3. Relations between homology and homotopy groups, Proc. Nat. Acad. Sci. U.S.A. vol. 29 (1943) pp. 155-158.

4. Relations between homology and homotopy groups of spaces, Ann. of Math. vol. 46 (1945) pp. 480-509.

P. HALL

1. A contribution to the theory of groups of prime power order, Proc. London Math. Soc. vol. 36 (1934) pp. 29-95.

2. Verbal and marginal subgroups, J. Reine Angew. Math. vol. 182 (1940) pp. 156-158.

H. HopF

1. Fundamentalgruppe und zweite Bettische Gruppe, Comment. Math. Helv. vol. 14 (1942)

F. LEVI pp. 257-309.

1. Über die Untergruppen der freien Gruppen. I, Math. Zeit. vol. 32 (1930) pp. 315-318.

2. Über die Untergruppen der freien Gruppen. II, Math. Zeit. vol. 37 (1933) pp. 90-97.

W. Magnus

1. Beziehungen zwischen Gruppen und Idealen in einem speziellen Ring, Math. Ann. vol. 111 (1935) pp. 259-280.

2. Allgemeine Gruppentheorie, Enzyklopaedie der mathematischen Wissenschaften, I, 4, 9, 2d. ed., 1939.

B. NEUMANN

1. Identical relations in groups. I, Math. Ann. vol. 114 (1937) pp. 506-525.

(48) See, for example, Baer [2]. 
O. SCHREIER

1. Über die Erweiterung von Gruppen. I, Monatshefte für Mathematik und Physik vol. 34 (1926) pp. 165-180.

2. Die Untergruppen der freien Gruppen, Hamburger Abhandlungen vol. 5 (1927) pp. 161183.

O, TeICHMÜLlER

1. Braucht der Algebraiker das Auswahlaxiom? Deutsche Mathematik vol. 4 (1939) pp. 567577.

H. Zassenhaus

1. Lehrbuch der Gruppentheorie. I, Hamburger Mathematische Einzelschriften vol. 21, 1937. M. ZORN

1. A remark on method in transfinite algebra, Bull. Amer. Math. Soc. vol. 41 (1935) pp. 667670.

UNIVERSITY OF ILLINOIS,

URBANA, ILL. 